ESAIM: COCV 22 (2016) 983-1016

DOI: $10.1051 / \mathrm{cocv} / 2016047$
ESAIM: Control, Optimisation and Calculus of Variations

www.esaim-cocv.org

\title{
CONTROL OF NON HOLONOMIC OR UNDER-ACTUATED MECHANICAL SYSTEMS: THE EXAMPLES OF THE UNICYCLE ROBOT AND THE SLIDER
}

\author{
BrigitTe D'AndRÉA-Novel ${ }^{1}$ AND SYlvain ThOREL ${ }^{2}$
}

\begin{abstract}
This paper is dedicated to the control of some classes of mechanical systems, not stabilizable by means of at least continuous state feedback laws. This is the case for non holonomic mechanical systems, an example being the unicycle robot, or for under-actuated mechanical systems, an example being the slider. The similarity between these two kinds of dynamical systems will be analyzed, with respect to controllability, stabilizability and flatness properties. The control design for slider stabilization could be viewed, to some extent, as an extension of the control law used for unicycle systems, but it is not so obvious if one wants to stabilize the slider at a chosen reference position, with a chosen fixed orientation. In fact, a new fixed point stabilization result will be given for the slider, and therefore, a switched control strategy will be proposed for both systems, based on differential flatness property to track moving non singular reference trajectories, and switching to periodic time-varying feedback laws to ensure final stabilization at rest equilibrium points. The method will be illustrated with success through simulation results and also first experimental results in the case of a terrestrial quadrotor tracking non singular reference trajectories.
\end{abstract}

Mathematics Subject Classification. 93B, 93C, 93D.

Received June 6, 2016. Accepted June 7, 2016.

\section{INTRODUCTION}

It is a great pleasure and honor to write this article in the special issue dedicated to Jean-Michel Coron. We would like to express our gratitude to Jean-Michel for his help, when working together on stabilization problems for nonlinear dynamical systems and also on boundary control of dynamical systems modeled by hyperbolic PDEs.

In the present paper, we are interested in non holonomic or under-actuated mechanical systems for which point stabilization cannot be achieved through continuous state feedback laws since they do not satisfy the necessary condition due to Brockett's theorem (see [5]). Thus the control design can be divided in two steps: one for non singular trajectory tracking purposes and the other for point stabilization. This approach will be illustrated through two examples: the first one is the so-called "unicycle" mobile robot which is a non holonomic vehicle,

\footnotetext{
Keywords and phrases. Non holonomic mechanical systems, under-actuated systems, fixed point stabilization, trajectory tracking, persistence of excitation condition, differential flatness property, time-varying feedback.

1 MINES ParisTech-CAOR, PSL, 60 Bvd St-Michel 75006 Paris, France. brigitte.dandrea-novel@mines-paristech.fr

2 SAGEM, 100 avenue de Paris 91344 Massy Cedex, France. sylvain.thorel@sagem.com
} 
and the second one is the "slider" which is an under-actuated mechanical system. The similarity between these two kinds of dynamical systems will be emphasized, with respect to controllability, stabilizability and flatness properties. Control laws stabilizing unicycle robots or sliders at a fixed point or towards exciting reference trajectories were largely investigated by the research community. Different strategies can be used to tackle the point stabilization issue. Time-varying control laws are very popular methods but discontinuous or practical stabilizations are alternatives. Due to their similarities, the control design for slider stabilization could be viewed, to some extent, as an extension of the control law used for unicycle systems. This is the case if one only wants to stop the slider, but it is not so obvious if one wants to stabilize it at a chosen reference position, with a chosen fixed orientation. In fact, a new fixed point stabilization result will be proposed for the slider, using a time-varying feedback law, inspired from [27] and which is similar, in some sense, to the "phantom tracking method" developed by Coron in [12].

In Section 2 we briefly recall modeling issues for these mechanical systems and we introduce the trajectory tracking problem. In Section 3 we study the structural controllability, stabilizability and differential flatness properties of the two systems and in Section 4 we propose our control design, namely: flatness-based control for trajectory tracking of non singular reference trajectories and time-varying feedback for fixed point stabilization of both systems. This method will be illustrated through simulation results and also first experimental results in the case of a terrestrial quadrotor tracking non singular reference trajectories. We conclude in Section 5.

\section{Modeling of Vehicles AND TRAJECTORY CONTROL PROBLEM}

The dynamical behaviors of non holonomic or under-actuated mechanical systems are recalled. Two examples are analyzed: the unicycle robot and the slider, for which the trajectory tracking problem is presented.

\subsection{Modeling}

The kinematic and dynamic model of a vehicle can be written in a canonical form as follows $[6,7]$ :

- Kinematic equation:

$$
\dot{q}=S(q) \eta
$$

- Dynamic equation:

$$
M(q) \dot{\eta}=-N(q, \eta) \eta+P(q, \eta, t)+C \tau .
$$

The vector $q \in \mathbb{R}^{n}$ contains the generalized configuration coordinates, the vector $\eta \in \mathbb{R}^{n-m}$ corresponds to the instantaneous non constrained velocities. The dimension of $\eta$ is the number of degrees of freedom of the vehicle. The vector $\tau \in \mathbb{R}^{p}$ contains the external forces or torques applied by the actuators to the system. The matrix $M(q) \in \mathbb{R}^{(n-m) \times(n-m)}$ is the invertible inertia matrix, $N(q, \eta) \eta$ is the $(n-m)$ vector of centrifugal and Coriolis torques and the vector $P(q, \eta, t) \in \mathbb{R}^{n-m}$ contains other terms such as gravity or frictious terms. The matrices $S(q) \in \mathbb{R}^{n \times(n-m)}$ and $C \in \mathbb{R}^{(n-m) \times p}$ have respective ranks $n-m$ and $p(n \geq n-m \geq p)$.

\subsubsection{Non holonomic vehicle}

Constraints linear in the velocities, which appear for example in rolling without slipping movements, restrict the space of instantaneous velocities but not necessarily the space of configurations. This is the case when the constraints are non holonomic, i.e. non integrable. Let us consider the following kinematic constraints:

$$
A^{\top}(q) \dot{q}=0,
$$

where $A^{\top}(q) \in \mathbb{R}^{m \times n}$ is of full rank $m$ and represents a set of $m$ independent velocity constraints. We suppose in the sequel that all the constraints are non holonomic, if this is not the case, the integrable constraints can be eliminated and the corresponding space configuration reduced. Let us point out that from (2.1), the matrix $S(q) \in \mathbb{R}^{n \times(n-m)}$ in equation (2.1) is such that $A^{\top}(q) S(q)=0$. Let us denote $\Delta(q)$ the distribution spanned by the $(n-m)$ vector fields $s_{j}(q), s_{j}$ being the $j$ th column of $S(q)$, and $\bar{\Delta}(q)$ the involutive closure of $\Delta(q)$. 


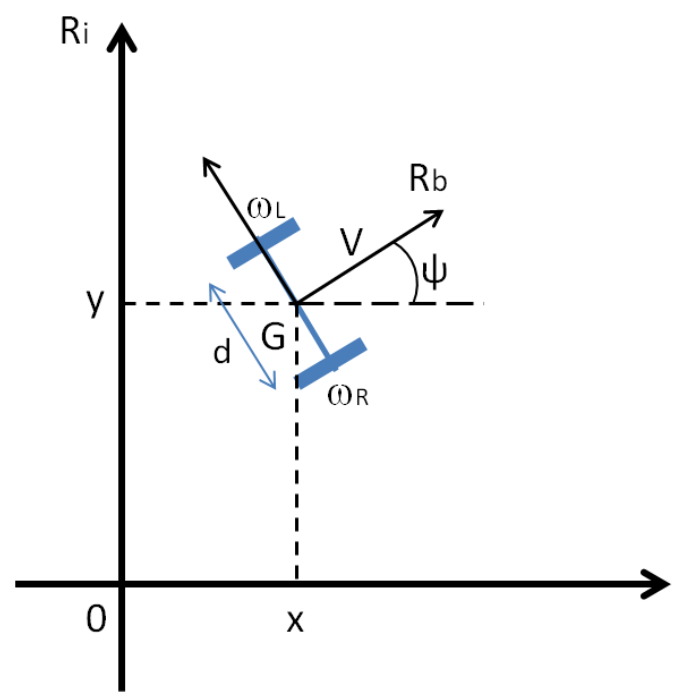

Figure 1. The unicycle robot.

Since all the $m$ constraints are supposed to be non integrable, it can be shown, due to Frobenius theorem, that $\bar{\Delta}(q)$ has dimension $n$, which means nothing but the fact that the LARC condition (Lie Algebra Rank Condition) is satisfied for the kinematic model (2.1) (see Def. 3.6), where $\eta$ is interpreted as the input control vector; therefore the kinematic model is strongly accessible from any configuration (see e.g. [35], Chap. 6, [26]). For a dynamical system without drift, strong accessibility implies controllability. Then, the kinematic equation of a non holonomic vehicle is controllable. Moreover, since $p=n-m$, the matrix $C$ is invertible, and the control vector $\tau$ can be easily chosen, for example to linearize the dynamic sub-system (2.2) so that the control of the global cascaded system (i.e. a system obtained by adding pure integrators to a previous sub-system) can then be handled using "backstepping" techniques (see e.g. $[25,40,46,51])$. This is the reason why one often considers only the kinematic model when studying non holonomic vehicles. Wheeled mobile robots satisfying both pure rolling and nonslipping conditions along the movements are undoubtedly the most popular non holonomic vehicles, and among them, the so-called unicycle robot which has been largely studied (for more details see e.g. $[2,3,6,7]$ ).

\section{The unicycle robot:}

This robot moves on the 2D-horizontal plane. The configuration vector $q=(x, y, \psi)^{\top}$ is of dimension $n=3$ and the vector $\eta=\left(v_{1}, \dot{\psi}\right)^{\top}$ has dimension 2. It is made of the longitudinal velocity $v_{1}$ and of the angular velocity $\dot{\psi}$. The $p=2$ physical control variables are the angular rotations $\omega_{R}$ and $\omega_{L}$ of the right and left wheels, see Figure 1. The third wheel is a free wheel which does not restrict the robot mobility. The non holonomic property of the unicycle comes from the fact that the robot cannot instantaneously move in the lateral direction, so that $p=n-m<n$. Due to the rolling without slipping assumption, the kinematic behavior of point $G$ can be easily obtained from $\omega_{R}, \omega_{L}$, the wheels' radius $R$ and the distance between the wheels $d$ :

$$
\left\{\begin{array}{l}
v_{1}=R\left(\omega_{R}+\omega_{L}\right) \\
\Omega=\frac{R}{2 d}\left(\omega_{R}-\omega_{L}\right) .
\end{array}\right.
$$

The kinematic equations of the unicycle robot can then be written:

$$
\left\{\begin{array}{l}
\dot{x}=v_{1} \cos (\psi) \\
\dot{y}=v_{1} \sin (\psi) \\
\dot{\psi}=\Omega .
\end{array}\right.
$$




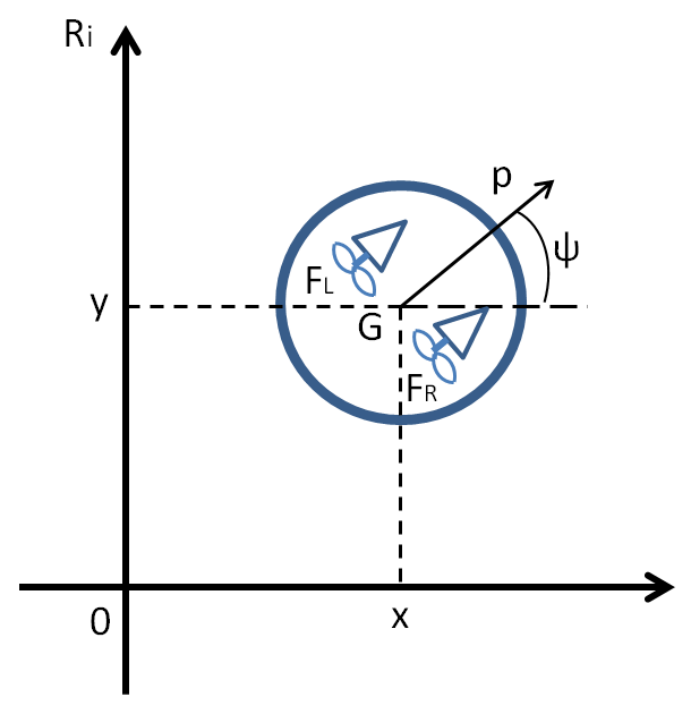

Figure 2. The slider.

\subsubsection{Under-actuated vehicles}

Moreoften, under-actuated vehicles have no restriction concerning the instantaneous velocities but are such that $p<n$. The whole dynamics (both kinematic and dynamic equations) has then to be considered. Except for wheeled mobile robots, most of the vehicles are under-actuated systems. The controls which are generally considered are twofold: firstly a thrust vector along the longitudinal axis and secondly an actuator to modify the orientation of the vehicle. The lateral axis is generally not directly actuated. Let us give some examples of such under-actuated vehicles (see e.g. [16]):

- marine vehicles: surface vessels (ships without lateral propellers), underwater vehicles, hovercrafts ...

- aerial vehicles: planes, unmanned aerial vehicles, drones ...

\section{The slider:}

The slider is an under-actuated vehicle, similar to marine vehicles (such as hovercrafts or surface vessels see e.g. [17]), or to terrestrial quadrotors such as the one studied in $[47,48]$. It moves on a $2 \mathrm{D}$ horizontal plane and has a configuration vector $q$ similar to the unicyle's one: $q=(x, y, \psi)^{\top}$ and $n=3$. But, the instantaneous velocities of vector $\eta$ are not restricted and $\eta$ has dimension $3: \eta=\left(v_{1}, v_{2}, \dot{\psi}\right)^{\top}$, where $v_{1}$ and $v_{2}$ are respectively the longitudinal and lateral velocities in the robot frame and $\dot{\psi}$ the angular velocity. Therefore, the control vector has dimension $p=2 \leq n=3$. This vehicle is described in Figure 2. It is actuated by two propellers producing forces $F_{L}$ and $F_{R}$. The sum of these two forces is directly linked to the acceleration of the vehicle, whereas the difference acts on the angular dynamics. Let us denote $\tau_{1}=F_{L}+F_{R}$ and $\tau_{2}=F_{R}-F_{L}$, the dynamics can be written:

$$
\left\{\begin{array}{l}
\dot{q}=S(q) \eta \\
m \dot{v_{1}}=m v_{2} \dot{\psi}+\tau_{1} \\
m \dot{v_{2}}=-m v_{1} \dot{\psi} \\
I \ddot{\psi}=\tau_{2},
\end{array}\right.
$$




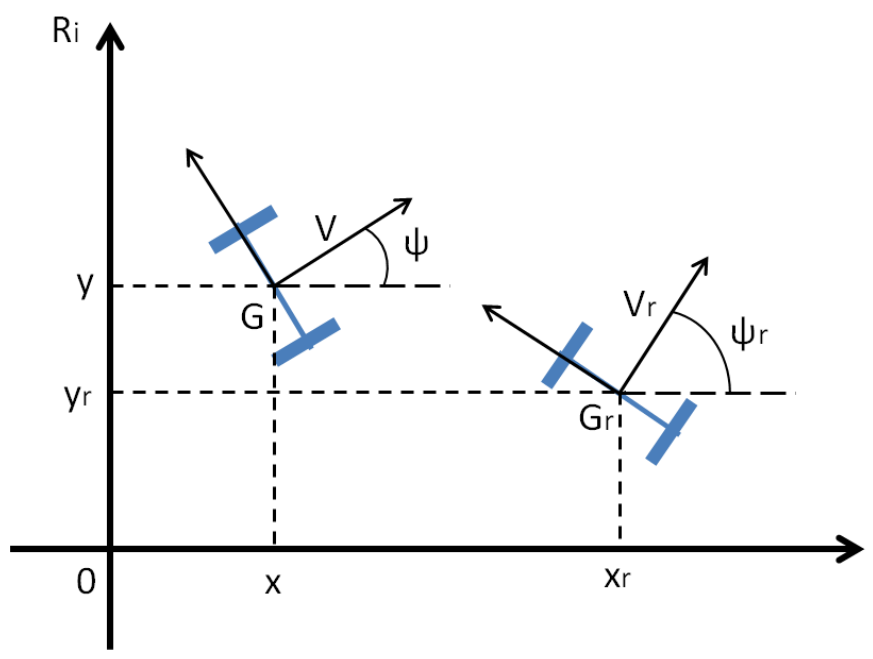

FIGURE 3. The trajectory tracking problem.

with

$$
S(q)=\left(\begin{array}{ccc}
C_{\psi} & -S_{\psi} & 0 \\
S_{\psi} & C_{\psi} & 0 \\
0 & 0 & 1
\end{array}\right)
$$

where $C_{\psi}$ and $S_{\psi}$ denote respectively $\cos (\psi)$ and $\sin (\psi), m$ being the vehicle mass, and $I$ the vehicle inertia. It should be noticed that friction terms have been neglected. Moreover, $S(q)$ is nothing but the rotation matrix from the inertial frame $R_{i}$ to the robot frame $R_{b}$. Therefore, equations (2.5) describe the dynamic behavior of the slider expressed in the vehicle frame and can be written as follows in the inertial frame:

$$
\left\{\begin{array}{l}
m \ddot{x}=\tau_{1} \cos (\psi) \\
m \ddot{y}=\tau_{1} \sin (\psi) \\
I \ddot{\psi}=\tau_{2} .
\end{array}\right.
$$

The similarity between these equations and the unicycle's ones (2.4) is then obvious.

\subsection{The trajectory tracking problem}

The aim of trajectory tracking displayed on Figure 3 is to make the robot's actual position and orientation asymptotically converge to the position and orientation of a reference virtual robot satisfying similar dynamical equations and constraints. The state variables of this reference robot are denoted with subscript $r$.

It is often simpler to express the error vector in the vehicle frame. Let us recall that a vector $[X]_{R_{i}}$ expressed in the frame $R_{i}$ and the same vector $[X]_{R_{b}}$ expressed in the robot frame $R_{b}$ are linked as follows, using the rotation matrix $R_{\psi}$ given below:

$$
[X]_{R_{b}}=R_{\psi}[X]_{R_{i}}
$$

with

$$
R_{\psi}=\left(\begin{array}{cc}
C_{\psi} & S_{\psi} \\
-S_{\psi} & C_{\psi}
\end{array}\right)
$$


Remark 2.1. Let us remember that the inverse of $R_{\psi}$ is equal to the transpose matrix of $R_{\psi}$ and that the time-derivative of $R_{\psi}$ can be easily computed as follows:

$$
\dot{R}_{\psi}=\dot{\psi} J R_{\psi}
$$

with

$$
J=\left(\begin{array}{cc}
0 & 1 \\
-1 & 0
\end{array}\right)
$$

\section{The unicycle robot:}

See $[7,22,45]$. In the unicycle case, the reference robot satisfies the following equations

$$
\left\{\begin{array}{l}
\dot{x}_{r}=v_{1 r} \cos \left(\psi_{r}\right) \\
\dot{y}_{r}=v_{1 r} \sin \left(\psi_{r}\right) \\
\dot{\psi}_{r}=\Omega_{r}
\end{array}\right.
$$

where $v_{1 r}$ and $\Omega_{r}$ are supposed to be bounded as well as their time-derivatives.

The objective is to find a control law $\left(v_{1}, \Omega\right)^{\top}=k\left(x, x_{r}, y, y_{r}, \psi, \psi_{r}, v_{1 r}, \Omega_{r}\right)$ such that the errors $e_{x}=x-x_{r}$, $e_{y}=y-y_{r}$ and $e_{\psi}=\psi-\psi_{r}$ asymptotically converge to 0 when $t$ tends to infinity.

The projection of the errors $e_{x}$ and $e_{y}$ in the robot frame will be denoted $e_{1}$ and $e_{2}$. The orientation error in the robot frame will be denoted $e_{3}$ but it is unchanged: $e_{\psi}=e_{3}$. The change of coordinates is then given by:

$$
\left(\begin{array}{l}
e_{1} \\
e_{2}
\end{array}\right)=R_{\psi}\left(\begin{array}{c}
e_{x} \\
e_{y}
\end{array}\right) ; e_{3}=e_{\psi}
$$

Using equations (2.4), (2.8), (2.9) and (2.10), the time-derivative of the error vector in the robot frame can be written as follows:

$$
\left(\begin{array}{c}
\dot{e_{1}} \\
\dot{e_{2}} \\
\dot{e_{3}}
\end{array}\right)=\left(\begin{array}{ccc}
0 & \dot{\psi} & 0 \\
-\dot{\psi} & 0 & 0 \\
0 & 0 & 0
\end{array}\right)\left(\begin{array}{l}
e_{1} \\
e_{2} \\
e_{3}
\end{array}\right)+\left(\begin{array}{c}
0 \\
\sin \left(e_{3}\right) \\
0
\end{array}\right) v_{1 r}+\left(\begin{array}{ll}
1 & 0 \\
0 & 0 \\
0 & 1
\end{array}\right)\left(\begin{array}{l}
u_{1} \\
u_{2}
\end{array}\right),
$$

where $u_{1}=v_{1}-v_{1 r} \cos \left(e_{3}\right)$ and $u_{2}=\Omega-\Omega_{r}$. Solving the trajectory tracking problem is then equivalent to finding a control law $\left(u_{1}, u_{2}\right)^{\top}$ making $\left(e_{1}, e_{2}, e_{3}\right)^{\top}$ asymptotically converge to $(0,0,0)^{\top}$.

\section{The slider:}

As for the unicycle case, let us introduce the error vector $\left(e_{x}, e_{y}, e_{\dot{x}}, e_{\dot{y}}, e_{\psi}, e_{\dot{\psi}}\right)^{\top}=\left(x-x_{r}, y-y_{r}, \dot{x}-\dot{x}_{r}, \dot{y}-\right.$ $\left.\dot{y}_{r}, \psi-\psi_{r}, \dot{\psi}-\dot{\psi}_{r}\right)^{\top}$, and its projection in the robot frame:

$$
\begin{aligned}
& \left(\begin{array}{l}
e_{1} \\
e_{2}
\end{array}\right)=R_{\psi}\left(\begin{array}{c}
e_{x} \\
e_{y}
\end{array}\right) ; e_{5}=e_{\psi}, \\
& \left(\begin{array}{l}
e_{3} \\
e_{4}
\end{array}\right)=R_{\psi}\left(\begin{array}{c}
e_{\dot{x}} \\
e_{\dot{y}}
\end{array}\right) ; e_{6}=e_{\dot{\psi}} .
\end{aligned}
$$

The virtual reference slider satisfies the following equations:

$$
\left\{\begin{array}{l}
m \ddot{x}_{r}=C_{\psi_{r}} \tau_{1 r} \\
m \ddot{y}_{r}=S_{\psi_{r}} \tau_{1 r} \\
I \ddot{\psi}_{r}=\tau_{2 r} .
\end{array}\right.
$$


As for the unicycle, using equations (2.6), (2.8), (2.12) and (2.13) leads to:

$$
\left(\begin{array}{c}
\dot{e_{1}} \\
\dot{e_{2}} \\
\dot{\dot{e}_{3}} \\
\dot{e_{4}} \\
\dot{e_{5}} \\
\dot{\dot{e}_{6}}
\end{array}\right)=\left(\begin{array}{cccccc}
0 & \dot{\psi} & 1 & 0 & 0 & 0 \\
-\dot{\psi} & 0 & 0 & 1 & 0 & 0 \\
0 & 0 & 0 & \dot{\psi} & 0 & 0 \\
0 & 0 & -\dot{\psi} & 0 & 0 & 0 \\
0 & 0 & 0 & 0 & 0 & 1 \\
0 & 0 & 0 & 0 & 0 & 0
\end{array}\right)\left(\begin{array}{c}
e_{1} \\
e_{2} \\
e_{3} \\
e_{4} \\
e_{5} \\
e_{6}
\end{array}\right)+\left(\begin{array}{c}
0 \\
0 \\
0 \\
\sin \left(e_{5}\right) \\
0 \\
0
\end{array}\right) u_{1 r}+\left(\begin{array}{ll}
0 & 0 \\
0 & 0 \\
1 & 0 \\
0 & 0 \\
0 & 0 \\
0 & 1
\end{array}\right)\left(\begin{array}{l}
u_{1}^{\prime} \\
u_{2}^{\prime}
\end{array}\right),
$$

with $u_{1 r}=\frac{\tau_{1 r}}{m}, u_{1}^{\prime}=\frac{1}{m}\left(\tau_{1}-\tau_{1 r} \cos \left(e_{3}\right)\right)$ and $u_{2}^{\prime}=\frac{1}{I}\left(\tau_{2}-\tau_{2 r}\right)$.

The objective is to find a control law $\left(\tau_{1}, \tau_{2}\right)^{\top}=k\left(x, x_{r}, y, y_{r}, \dot{x}, \dot{x}_{r}, \dot{y}, \dot{y}_{r}, \psi, \psi_{r}, \dot{\psi}, \dot{\psi}_{r}, \tau_{1_{r}}, \tau_{2 r}\right)$ such that the error vector $\left(e_{x}, e_{y}, e_{\dot{x}}, e_{\dot{y}}, e_{\psi}, e_{\dot{\psi}}\right)^{\top}$ asymptotically converges to 0 when $t$ tends to infinity.

\section{Controllability, Stabilizability And Differential Flatness Properties}

The controllability properties of the two systems are analyzed, first for the tangent linearized control systems and then in terms of STLC (Small Time Local Controllability) for the original ones. Then, stabilizability properties are deduced. Finally, the structural "differential flatness property" is studied for both systems.

Before that, let us briefly recall some definitions and properties concerning STLC for a time-invariant nonlinear dynamical system $\Sigma$ with state vector $X$ and control input vector $u$ :

$$
\Sigma: \dot{X}=f(X, u) .
$$

Definition 3.1 ([13], p. 125). Let $\left(X_{e}, u_{e}\right) \in \mathcal{O}\left(\mathcal{O}\right.$ an open subset of $\left.\mathbb{R}^{n}\right)$, an equilibrium point of (3.1). This system is "small-time locally controllable", or STLC at $\left(X_{e}, u_{e}\right)$ if, for every real number $\epsilon>0$, there exists a real number $\eta>0$ such that, for every $X_{0} \in B_{\eta}\left(X_{e}\right)=\left\{X \in \mathbb{R}^{n} ;\left|X-X_{e}\right|<\eta\right\}$ and for every $X_{1} \in B_{\eta}\left(X_{e}\right)$, there exists a measurable function $u:[0, \epsilon] \rightarrow \mathbb{R}^{m}$ such that

$$
\begin{gathered}
\left|u(t)-u_{e}\right| \leq \epsilon, \quad \forall t \in[0, \epsilon], \\
\left(\dot{X}=f(X, u(t)), X(0)=X_{0}\right) \Rightarrow\left(X(\epsilon)=X_{1}\right) .
\end{gathered}
$$

One does not know any checkable necessary and sufficient condition for STLC for a general system $\Sigma$. However, some powerful necessary conditions are known and will be detailed below.

Definition 3.2. Let $\left(X_{e}, u_{e}\right)$ be an equilibrium point of system $\Sigma$. The tangent linearized control system at $\left(X_{e}, u_{e}\right)$ is the following linear dynamical system:

$$
\dot{\delta}_{x}=\frac{\partial f}{\partial x}\left(X_{e}, u_{e}\right) \delta_{x}+\frac{\partial f}{\partial u}\left(X_{e}, u_{e}\right) \delta_{u}=A \delta_{x}+B \delta_{u},
$$

where $\delta_{x}=X-X_{e}, \delta_{u}=u-u_{e}, X(t) \in \mathbb{R}^{n}$ and $u(t) \in \mathbb{R}^{m}$.

The controllability property of a time-invariant linear system can be checked using the so-called Kalman criterion (see e.g. [1], Chap. 5).

Theorem 3.3 ([1], p. 102). Let $\left(X_{e}, u_{e}\right)$ be an equilibrium point of system $\Sigma$. The tangent linearized control system $(3.2)$ at $\left(X_{e}, u_{e}\right)$ is controllable, if and only if the rank of the controllability matrix $\mathcal{C}=$ $\left(\begin{array}{lllll}B & A B & A^{2} B & \ldots & A^{n-1} B\end{array}\right)$ is equal to $n=\operatorname{dim}(X)$, the order of the system.

Theorem 3.4 ([13], p. 128). Let $\left(X_{e}, u_{e}\right)$ be an equilibrium point of system $\Sigma$. Let us suppose that the tangent linearized control system (3.2) at $\left(X_{e}, u_{e}\right)$ is controllable, then $\Sigma$ is STLC at $\left(X_{e}, u_{e}\right)$.

If the tangent linearized control system is not controllable, one should however test the "Lie Algebra Rank Condition" denoted LARC, which relies on iterated Lie brackets, recalled below. 
Definition 3.5. Let $\Omega$ be an open subset of $\mathbb{R}^{n}$ and $\mathcal{F}$ a family of $C^{\infty}$ vector fields in $\Omega$. The notation $\operatorname{Lie}(\mathcal{F})$ is the Lie algebra generated by the vector fields $\mathcal{F}$, or in other words, the smallest linear subspace $E$ of $C^{\infty}\left(\Omega ; \mathbb{R}^{n}\right)$ satisfying the following two conditions:

$$
\begin{gathered}
\mathcal{F} \subset E, \\
(X \in E \text { and } Y \in E) \Rightarrow([X, Y] \in E) .
\end{gathered}
$$

Let us denote $\mathcal{A}\left(X_{e}, u_{e}\right)=\left\{g\left(X_{e}\right) ; g \in \operatorname{Lie}\left(\frac{\partial^{|\alpha|} f}{\partial u^{\alpha}}\left(., u_{e}\right), \alpha \in \mathbb{N}^{m}\right)\right\} \cdot \mathcal{A}\left(X_{e}, u_{e}\right)$ can be obtained by considering the control vector fields and their iterated Lie brackets (cf. [13], Chap. 3.2).

Definition 3.6 (LARC). The system $\Sigma$ satisfies the LARC at the equilibrium point $\left(X_{e}, u_{e}\right)$ if

$$
\mathcal{A}\left(X_{e}, u_{e}\right)=\mathbb{R}^{n} .
$$

Theorem 3.7. Let us suppose that the system $\Sigma$ is $S T L C$ at the equilibrium point $\left(X_{e}, u_{e}\right)$ and that $f$ is analytic. Then $\Sigma$ satisfies the LARC at $\left(X_{e}, u_{e}\right)$.

The converse is not true in general except for linear time-invariant dynamical systems for which the LARC is equivalent to the Kalman controllability criterion (see Thm. 3.3) or for driftless affine control dynamical systems, as detailed in the following theorem.

Theorem 3.8 (Rashevski-Chow [13], p. 135). Let $\Omega$ be an open subset of $\mathbb{R}^{n}, \Omega \times\{0\} \subset \mathcal{O}$. Let us assume that there exist $f_{1}, \ldots, f_{m} \in C^{\infty}\left(\Omega ; \mathbb{R}^{n}\right)$, such that

$$
f(X, u)=\sum_{i=1}^{m} u_{i} f_{i}(X), \quad \forall(X, u) \in \mathcal{O} .
$$

Let $X_{e} \in \Omega$ such that

$$
\mathcal{A}\left(X_{e}, 0\right)=\mathbb{R}^{n} .
$$

Then, the control system $\dot{X}=f(X, u)$ is $S T L C$ at the equilibrium point $\left(X_{e}, 0\right) \in \mathcal{O}$.

Remark 3.9. Except for driftless control affine dynamical systems, the LARC condition is not sufficient to prove local controllability. Sussmann's theorem, recalled in ([13], p. 143), gives, in addition to LARC, some necessary conditions using so-called "Bad and Good iterated Lie brackets" as detailed in ([13], pp. 141-149).

Remark 3.10. Note that if reference trajectories are reduced to equilibrium points in the case of trajectory tracking problems, systems (2.11) and (2.14) can be seen as time-varying linear systems, since the state matrix depends on $t$ through $\dot{\psi}(t)$. In that case, some stabilization results exist in the case of slowly varying persistently exciting signals. Let us give the corresponding result in the following proposition which can be easily deduced from ([24], Thm. 2).

Proposition 3.11. Let $A: \mathbb{R} \rightarrow \mathbb{R}^{n \times n}$ be of class $C^{1}$ and let $B$ a real matrix of size $n \times m$. We assume that for every $s \in \mathbb{R}^{*}$, the pair $(A(s), B)$ is controllable. Let $T>0, M>0$ and $\epsilon>0$. Then there exist $\delta_{1}>0$, $\delta_{2}>0$ such that for every T-periodic Lipschitz function $\phi(t)$ satisfying:

- (i) $|\phi(t)| \leq M$ for all $t \geq 0$;

- (ii) $\left|\phi(t)-\phi\left(t^{\prime}\right)\right| \leq \delta_{2}\left|t-t^{\prime}\right|$ for all $t, t^{\prime} \geq 0$;

- (iii)

$$
\forall t \geq 0, \exists s: t-\delta_{1} \leq s \leq t \text { such that }|\phi(t)| \geq \epsilon
$$

then the system $\Sigma$ is controllable on every time interval of length at least equal to $2 T$, where $\Sigma$ is the following linear time-varying system:

$$
\Sigma: \quad \dot{X}=A(\phi(t)) X+B u .
$$

This result will be used in the context of point stabilization of the slider in Section 4.2.2.

Definition 3.12. The condition (iii) on the function $\phi(t)$ is known as "persistence of excitation condition". 


\subsection{Tangent linearized control systems}

\section{The unicycle robot:}

Let us consider the equilibrium point $(e=0, u=0)$, the tangent linearized system (3.4) of (2.11) can be written:

$$
\left(\begin{array}{c}
\dot{e_{1}} \\
\dot{e_{2}} \\
\dot{e_{3}}
\end{array}\right)=\left(\begin{array}{ccc}
0 & \dot{\psi}_{r} & 0 \\
-\dot{\psi}_{r} & 0 & v_{1 r} \\
0 & 0 & 0
\end{array}\right)\left(\begin{array}{l}
e_{1} \\
e_{2} \\
e_{3}
\end{array}\right)+\left(\begin{array}{ll}
1 & 0 \\
0 & 0 \\
0 & 1
\end{array}\right)\left(\begin{array}{l}
u_{1} \\
u_{2}
\end{array}\right) .
$$

In our case, $n=3$ and $\mathcal{C}$ is given by:

$$
\mathcal{C}=\left(\begin{array}{cccccc}
1 & 0 & 0 & 0 & -\dot{\psi}_{r}^{2} & v_{1 r} \dot{\psi}_{r} \\
0 & 0 & -\dot{\psi}_{r} & v_{1 r} & 0 & 0 \\
0 & 1 & 0 & 0 & 0 & 0
\end{array}\right)
$$

This controllability matrix is clearly of full rank if and only if $\left(v_{1 r}, \dot{\psi}_{r}\right) \neq(0,0)$. In other words, the tangent linearized system of the unicycle robot (3.4) is not controllable at equilibrium points. But, from Theorem 3.4, it is known that the nonlinear system $(2.11)$ is STLC if the reference trajectory is such that $\left(v_{1 r}, \dot{\psi}_{r}\right) \neq(0,0)$.

\section{The slider:}

As for the unicycle, the Kalman criterion is applied to the tangent linearized control system of the slider at an equilibrium point $\left(e=0, u^{\prime}=0\right)$ which has the following form:

$$
\left(\begin{array}{c}
\dot{e_{1}} \\
\dot{e_{2}} \\
\dot{e_{3}} \\
\dot{e_{4}} \\
\dot{\dot{e}_{5}} \\
\dot{e_{6}}
\end{array}\right)=\left(\begin{array}{cccccc}
0 & \dot{\psi_{r}} & 1 & 0 & 0 & 0 \\
-\dot{\psi_{r}} & 0 & 0 & 1 & 0 & 0 \\
0 & 0 & 0 & \dot{\psi}_{r} & 0 & 0 \\
0 & 0 & -\dot{\psi}_{r} & 0 & u_{1 r} & 0 \\
0 & 0 & 0 & 0 & 0 & 1 \\
0 & 0 & 0 & 0 & 0 & 0
\end{array}\right)\left(\begin{array}{l}
e_{1} \\
e_{2} \\
e_{3} \\
e_{4} \\
e_{5} \\
e_{6}
\end{array}\right)+\left(\begin{array}{ll}
0 & 0 \\
0 & 0 \\
1 & 0 \\
0 & 0 \\
0 & 0 \\
0 & 1
\end{array}\right)\left(\begin{array}{l}
u_{1}^{\prime} \\
u_{2}^{\prime}
\end{array}\right) .
$$

It can be easily checked that the rank of the associated controllability matrix $\mathcal{C}$ is equal to $n=6$ when $\left(u_{1_{r}}, \dot{\psi}_{r}\right) \neq(0,0)$. Then, the tangent linearized control system of the slider is not controllable when $\left(u_{1_{r}}, \dot{\psi}_{r}\right)=$ $(0,0)$, or in other words, for trajectories such that the longitudinal acceleration and the angular velocity are zero. As for the unicycle, the original nonlinear system is however STLC when $\left(u_{1_{r}}, \dot{\psi}_{r}\right) \neq(0,0)$ from Theorem 3.4.

Remark 3.13. If one had added viscous friction terms as for example in [36], a trajectory satisfying $\left(u_{1_{r}}, \dot{\psi}_{r}\right)=$ $(0,0)$ would be nothing but a fixed equilibrium point.

\subsection{The STLC property}

The non controllability of the tangent linearized control system at a fixed point does not necessarily imply that the original nonlinear system does not satisfy the STLC property. In fact, we will show that the LARC is satisfied for both the unicycle and the slider, as well as the STLC property.

\section{The unicycle robot:}

The following equations (3.6) are nothing but those of (2.11) in the particular case of a fixed point reference trajectory $\left(v_{1 r}, \dot{\psi}_{r}\right)=(0,0)$.

$$
\underbrace{\left(\begin{array}{c}
\dot{e_{1}} \\
\dot{e_{2}} \\
\dot{e_{3}}
\end{array}\right)}_{\dot{e}}=\underbrace{\left(\begin{array}{c}
0 \\
-e_{1} \\
1
\end{array}\right)}_{X_{1}} \Omega+\underbrace{\left(\begin{array}{l}
1 \\
0 \\
0
\end{array}\right)}_{X_{2}} U_{1} \text { with } U_{1}=v_{1}+e_{2} \Omega .
$$


The Lie bracket $\left[X_{1}, X_{2}\right]$ is computed as follows:

$$
\left[X_{1}, X_{2}\right]=\underbrace{\frac{\partial X_{2}}{\partial e}}_{0} X_{1}-\frac{\partial X_{1}}{\partial e} X_{2}=-\left(\begin{array}{ccc}
0 & 0 & 0 \\
-1 & 0 & 0 \\
0 & 0 & 0
\end{array}\right) X_{2}=\left(\begin{array}{l}
0 \\
1 \\
0
\end{array}\right) .
$$

Then the unicycle satisfies the LARC since

$$
\begin{aligned}
& \mathcal{A}\left(\left(v_{1 r}, \dot{\psi}_{r}\right)=(0,0)\right)=\left\{X_{1}, X_{2},\left[X_{1}, X_{2}\right]\right\} \\
& =\left\{\left(\begin{array}{c}
0 \\
-e_{1} \\
1
\end{array}\right),\left(\begin{array}{l}
1 \\
0 \\
0
\end{array}\right),\left(\begin{array}{l}
0 \\
1 \\
0
\end{array}\right)\right\}=\mathbb{R}^{3} .
\end{aligned}
$$

Moreover, system (3.6) being a driftless control affine system, and the vector fields being analytic, the unicycle is STLC for fixed point trajectories from Theorem 3.8.

\section{The slider:}

Since the slider dynamics in the case $\left(u_{1 r}, \dot{\psi}_{r}\right)=(0,0)$, with added viscous friction terms given by (3.7) is quite similar to the one of the under-actuated surface vessel studied in [36] $\left(d_{1}, d_{2}, d_{3}\right.$ being strictly positive friction coefficients), we conclude that the LARC is satisfied for the slider (the assumption of viscous friction terms is not restrictive, but the heavy computation of the iterated Lie brackets is not detailed in the present paper).

$$
\begin{gathered}
\left(\begin{array}{c}
\dot{e_{1}} \\
\dot{e_{2}} \\
\dot{e_{3}} \\
\dot{e_{4}} \\
\dot{e_{5}} \\
\dot{e_{6}}
\end{array}\right)=f(e)+X_{1} u_{1}^{\prime}+X_{2} u_{2}^{\prime} \text { with } \\
f(e)=\left(\begin{array}{cccccc}
0 & e_{6} & 1 & 0 & 0 & 0 \\
-e_{6} & 0 & 0 & 1 & 0 & 0 \\
0 & 0 & -\frac{d_{1}}{m} & e_{6} & 0 & 0 \\
0 & 0 & -e_{6} & -\frac{d_{2}}{m} & 0 & 0 \\
0 & 0 & 0 & 0 & 0 & 1 \\
0 & 0 & 0 & 0 & 0 & -\frac{d_{3}}{I}
\end{array}\right) e, X_{1}=\left(\begin{array}{l}
0 \\
0 \\
1 \\
0 \\
0 \\
0
\end{array}\right), X_{2}=\left(\begin{array}{l}
0 \\
0 \\
0 \\
0 \\
0 \\
1
\end{array}\right) .
\end{gathered}
$$

From [36],

$$
\begin{gathered}
\mathcal{A}=\left\{X_{1}, X_{2},\left[f, X_{1}\right],\left[f, X_{2}\right],\left[X_{2},\left[f, X_{1}\right]\right],\right. \\
\left.\left[X_{2},\left[\left[X_{1}, f\right], f\right]\right]\right\}=\mathbb{R}^{6} .
\end{gathered}
$$

The vector fields $f, X_{1}, X_{2}$ being analytic, the LARC is then satisfied. Contrarily to the unicycle case, the slider dynamics has a drift term and the LARC is not sufficient to prove the local controllability. However, it has been shown in [36], that system (3.7) satisfies Sussmann's theorem mentioned in Remark 3.9, which implies that the slider is STLC at every fixed equilibrium point (see Rem. 3.13).

To conclude, STLC is satisfied for the two systems at each trajectory $\left(v_{1 r}, \dot{\psi}_{r}\right) \in \mathbb{R}^{2}$ respectively $\left(u_{1 r}, \dot{\psi}_{r}\right) \in \mathbb{R}^{2}$.

\subsection{Stabilizability properties}

Since the tangent linearized systems of both the unicycle and the slider are controllable at each exciting reference trajectory, respectively $\left(v_{1 r}, \dot{\psi}_{r}\right) \neq(0,0)$ and $\left(u_{1 r}, \dot{\psi}_{r}\right) \neq(0,0)$, it is then possible to find continuous state feedback laws asymptotically stabilizing such reference trajectories. However, the elaboration of an asymptotically stabilizing feedback law for a fixed equilibrium point is more difficult and even impossible by means of continuous state feedback laws, as shown from Brockett's Theorem 3.14 recalled below. 
Theorem 3.14 (Brockett [5]). If system $\Sigma: \dot{X}=f(X, u)$ can be locally asymptotically stabilized at the origin $(X=0, u=0)$ by a continuous state feedback law, then the image by $f$ of any neighborhood of $(0,0) \in \mathbb{R}^{n} \times \mathbb{R}^{m}$ is a neighborhood of $0 \in \mathbb{R}^{n}$.

Remark 3.15. The unicycle robot and the slider are not stabilizable at fixed equilibrium points by means of at least continuous state feedback laws. Let us consider a neighborhood of the origin of the unicycle $\left\{e=(0, \epsilon, 0)^{\top}\right\}$ with $\epsilon>0$; it clearly does not belong to the image of $\left\{f=\left(U_{1},-e_{1} \Omega, \Omega\right)^{\top}\right\}$ defined at equation (3.6). In the same way, the neighborhood of the origin of the slider $\left\{e=(\epsilon, 0,0,0,0,0)^{\top}\right\}$ does not belong to the image of the slider dynamics given by (3.7).

Consequently, fixed point stabilization needs other control approaches than classical continuous state feedback laws which allow stabilization of exciting reference trajectories. Among those control methods one can cite:

- smooth time-varying state feedback laws, moreoften time-periodic;

- discontinuous state feedback laws;

- control laws ensuring practical stabiliy, i.e. the stabilization of a small neighborhood containing the equilibrium point.

Concerning time-varying feedback laws, Coron has shown in [10] that global asymptotic stabilization of the origin of a STLC driftless affine control system could be achieved through smooth time-periodic state feedback laws (see also [13], Chap. 11, Thm. 11.14). Moreover, this result can be extended to local asymptotic stabilization of the origin of STLC general nonlinear control system $\Sigma: \dot{X}=f(X, u)$ using time-varying state feedback laws (see [13], Chap. 11, Thm. 11.28), [11]. In that context, many authors have proposed stabilizing time-varying feedback laws, see for example $[10,30,31,43,44]$ in the case of chained systems (see e.g. [34] for this notion) and also [14] for fixed point stabilization of the unicycle. See also [36,37] for stabilization of under-actuated surface vessels.

Some authors used invariant discontinuous control laws to stabilize under-actuated vehicles to a fixed point. We can cite for example [41] where conditions on the initial state must be satisfied to ensure the convergence. An alternative was proposed and described in [17]. In this paper, there is no constraint on the initial state but the yaw angle is not imposed to converge to zero.

Control laws ensuring a perfect tracking of an admissible trajectory, which is of exciting type or a fixed point, are not easy to design. This limitation leads authors to consider alternatives like practical approaches, the idea being to stabilize the system in an arbitrary small neighborhood of the reference trajectory (see for example [38] where the authors use averaging and backstepping techniques to obtain global practical stabilization for an under-actuated ship). Obviously, this kind of method does not allow asymptotic stabilization but offer other advantages. It can be possible to stabilize a vehicle on non admissible reference trajectories and in the same time, it can also facilitate the trajectory generation process. An approach illustrating this practical concept is for example the tranverse function method (see $[32,33]$ for details).

\subsection{Differential flatness property}

As already mentioned, differential flatness property will allow both mechanical systems to track moving non singular reference trajectories, as it will be explained in Section 4.1. Let us now briefly recall some definitions concerning differential flatness.

Definition 3.16 (see [19]). The system $\Sigma$, affine in the control, defined by:

$$
\dot{X}=f(X)+\sum_{i=1}^{m} g_{i}(X) u_{i}, \quad X \in \mathbb{R}^{n}, \quad u \in \mathbb{R}^{m}
$$

is said to be differentially flat if there exist an $m$-uple $Y=\left(Y_{1}, \ldots, Y_{m}\right)$, a function $h: \mathbb{R}^{n} \times\left(\mathbb{R}^{m}\right)^{r+1} \rightarrow \mathbb{R}^{m}$, a function $\phi:\left(\mathbb{R}^{m}\right)^{r} \rightarrow \mathbb{R}^{n}$ and $\psi:\left(\mathbb{R}^{m}\right)^{r+1} \rightarrow \mathbb{R}^{m}$ such that:

$$
\begin{gathered}
Y=h\left(X, u, \dot{u}, \ldots, u^{(r)}\right), \\
X=\phi\left(Y, \dot{Y}, \ldots, Y^{(r-1)}\right), \\
u=\psi\left(Y, \dot{Y}, \ldots, Y^{(r-1)}, Y^{(r)}\right) .
\end{gathered}
$$

The components $Y_{i}$ of $Y$ are called the flat outputs of the system. 
It is interesting to point out that the whole system's dynamic behavior is reduced to the behavior of its flat outputs. Finding such flat outputs is then an easy way to generate reference trajectories and elaborate dynamic linearizing feedback laws such that the tracking errors exponentially converge to zero. Trajectory tracking using the flatness property will be studied both for the unicycle robot and the slider in Section 4.1.

\section{Control Design}

As we have seen in the previous section, due to Brockett's Theorem 3.14, it is impossible to stabilize fixed equibrium points through continuous feedback laws for both systems. However, tracking moving reference trajectories seems to be easier. So, we propose in this section to solve the posture tracking problem in two steps: first, we design a flatness-based control law to make the robot exponentially converge to a nonsingular (i.e. moving) reference trajectory and then we achieve final fixed point stabilization using a time-varying (periodic) feedback law.

\subsection{Trajectory tracking via a differential flatness approach}

We consider successively the case of the unicycle and the slider.

\subsubsection{The unicycle robot}

Let us first consider the two first equations of the unicycle robot (2.4), giving the first time-derivative of $x$ and $y$. It can be seen that the only input which acts on $\dot{x}$ and $\dot{y}$ is $v_{1}$. So, in the framework of exact feedback linearization (see e.g. [21]), $x$ and $y$ can be considered as "output functions", and the corresponding so-called decoupling matrix $\Delta$ is therefore singular since:

$$
\left(\begin{array}{c}
\dot{x} \\
\dot{y}
\end{array}\right)=\left(\begin{array}{cc}
\cos (\psi) & 0 \\
\sin (\psi) & 0
\end{array}\right)\left(\begin{array}{c}
v_{1} \\
\Omega
\end{array}\right)=\Delta\left(\begin{array}{c}
v_{1} \\
\Omega
\end{array}\right) .
$$

We apply the idea of the so-called "dynamic extension algorithm" (see e.g. $[8,15]$ ). The principle of this algorithm applied to system $\Sigma$ is to delay some "combinations of inputs" simultaneously affecting several outputs, via the addition of integrators, in order to enable other inputs to act in the meanwhile and therefore hopefully to obtain an extended linear and decoupled closed-loop system of the form:

$$
Y_{k}^{\left(r_{k}\right)}=w_{k}, k=1, \ldots, m
$$

where $Y_{k}^{(i)}$ denotes the $i$ th time-derivative of the $k$ th output $Y_{k}, r_{k}$ is called the relative degree of $Y_{k}$ and $w_{k}$ are the new auxiliary inputs. Let us recall the definition of the decoupling matrix $\Delta$ :

$$
\Delta_{i j}=\frac{\partial Y_{i}^{\left(r_{i}\right)}}{\partial u_{j}}, i, j=1, \ldots, m .
$$

The corresponding dynamic feedback has the following form:

$$
\left\{\begin{array}{l}
u=\alpha(X, \chi, w) \\
\dot{\chi}=a(X, \chi, w)
\end{array}\right.
$$

$\chi$ being the added state and $w$ the auxiliary input vector. To get full linearization we must have:

$$
\sum_{i=1}^{m} r_{i}=\bar{n},
$$

where $\bar{n}$ is the dimension of the extended state vector $\bar{X}=(X, \chi)^{\top}$. 
Definition 4.1. If (4.5) is satisfied, the corresponding functions $Y_{i}=h_{i}(X), i=1, \ldots, m$ are called "linearizing outputs".

Moreover, if (4.5) is satisfied, then:

$$
\zeta=\Psi(\bar{X})=\left(Y_{1}, \ldots, Y_{1}^{\left(r_{1}-1\right)}, \ldots, Y_{m}, \ldots, Y_{m}^{\left(r_{m}-1\right)}\right)^{\top}
$$

is a local diffeomorphism (see $[8,9]$ ). From the linearization point of view, the control law becomes singular when the determinant of the square decoupling matrix is zero. Generically, this corresponds in the extended state-space to a $(\bar{n}-1)$-dimensional submanifold. From [8], we know that this submanifold contains the domain where the diffeomorphism $\Psi(\bar{X})$ is singular. Consequently, at any point where the decoupling matrix is not singular, the diffeomorphism $\Psi(\bar{X})$ exists.

When full linearization is obtained (at least generically), we can easily achieve reference trajectory tracking with stability. More precisely, let $X_{r}(t)$ be a smooth reference trajectory satisfying the system dynamical equations, then according to Definition 4.1 we have:

$$
\left(Y_{r}\right)_{i}=h_{i}\left(X_{r}\right), i=1, \ldots, m
$$

and according to $(4.6) \zeta_{r}(t)$ is defined as follows:

$$
\zeta_{r}=\left(\left(Y_{r}\right)_{1}, \ldots,\left(Y_{r}\right)_{1}^{\left(r_{1}-1\right)}, \ldots,\left(Y_{r}\right)_{m}, \ldots,\left(Y_{r}\right)_{m}^{\left(r_{m}-1\right)}\right)^{\top}
$$

This implies that:

$$
\bar{X}_{r}=\Psi^{-1}\left(\zeta_{r}\right) .
$$

The following proposition shows how to select the auxiliary control inputs $w_{i}$ in (4.2) in order to achieve the tracking with stability of $\zeta_{r}(t)$ and consequently of $X_{r}(t)$.

Proposition 4.2. The following auxiliary control laws $w_{i}, i=1, \ldots, m$ :

$$
w_{i}=\left(Y_{r}\right)_{i}^{\left(r_{i}\right)}+\sum_{j=0}^{r_{i}-1} \alpha_{i}^{j}\left(Y_{i}^{(j)}-\left(Y_{r}\right)_{i}^{(j)}\right)
$$

generically ensure that the trajectory error vector $\tilde{\zeta}=\zeta-\zeta_{r}$ exponentially converges to zero, provided that the arbitrary constants $\alpha_{i}^{j}$ are suitably chosen. Moreover, $\tilde{X}=X-X_{r}$ also asymptotically converges to zero.

Proof. The exponential convergence of $\tilde{\zeta}$ to zero is easy to prove, provided a suitable choice of the constants $\alpha_{i}^{j}$ which will determine the exponential rate of convergence of the error vector $\tilde{\zeta}$. Moreover, $\Psi$ being a diffeomorphism, this implies that $\bar{X}$ tends to $\bar{X}_{r}$ and therefore that $X$ asymptotically tends to $X_{r}$.

Remark 4.3. The problem of finding sufficient conditions for dynamic feedback linearization is open, and similar to the problem of finding flat output functions since flatness is equivalent to dynamic linearizability by endogeneous compensators. A dynamic compensator of the form (4.4) is endogeneous if $\chi$ can be expressed as a function of $x, u$ and a finite number of time-derivatives of $u$ (for details see $[4,18,19]$ ). Moreover, linearizing output functions are also flat outputs. One can also refer to [42] for necessary conditions for dynamic feedback linearization.

For the unicycle robot, considering (4.1) shows that the input $v_{1}$ has to be delayed to hopefully make the second input $\Omega$ appear in the second order time-derivatives of $x$ and $y$. Let us then introduce the added state $\chi_{1}=v_{1}$ and the new input $U_{1}$ (which is nothing but the time-derivative of $v_{1}$ ):

$$
\dot{v}_{1}=U_{1} .
$$


Then, computing $\ddot{x}$ and $\ddot{y}$, the extended system with extended vector $\bar{X}=\left(x, y, \psi, \chi_{1}\right)^{\top}$ is now fully linearizable by means of static state feedback with new input vector $\left(U_{1}, \Omega\right)^{\top}$ and diffeomorphism:

$$
\Psi(\bar{X})=(x, y, \dot{x}, \dot{y})^{\top} .
$$

More precisely, we have:

$$
\left(\begin{array}{c}
\ddot{x} \\
\ddot{y}
\end{array}\right)=\left(\begin{array}{cc}
\cos (\psi) & -\sin (\psi) \chi_{1} \\
\sin (\psi) & \cos (\psi) \chi_{1}
\end{array}\right)\left(\begin{array}{c}
U_{1} \\
\Omega
\end{array}\right)=\Delta\left(\begin{array}{c}
U_{1} \\
\Omega
\end{array}\right),
$$

and the new decoupling matrix $\Delta$ is generically invertible. The only singularity corresponds to $\operatorname{det}(\Delta)=\chi_{1}=0$. It means that the longitudinal velocity of the unicycle, $\chi_{1}=v_{1}$, cannot be zero, or in other words, as expected, that the robot must not stop. For more details see also $[2,3]$.

As mentioned in Remark 4.3, let us check in the following proposition that $x$ and $y$ are flat outputs for the unicycle robot (2.4).

Proposition 4.4. The unicycle robot (2.4) is flat with outputs $Y_{1}=x$ and $Y_{2}=y$.

Proof. Using (2.4), it can be easily seen that:

$$
\psi=\arctan \left(\frac{\dot{y}}{\dot{x}}\right) \text { and } v_{1}= \pm \sqrt{\dot{x}^{2}+\dot{y}^{2}} .
$$

It results that $\Omega=\dot{\psi}$ can be written as a function of $(\dot{x}, \dot{y}, \ddot{x}, \ddot{y})$, which means that $x$ and $y$ are flat outputs and concludes the proof.

The important drawback of exact linearization by feedback lies in the fact that it is not applicable around the singularities of the decoupling matrix $\Delta$, i.e. around rest positions as we have seen for the unicycle robot. Nevertheless, we can show that it can be implemented in practical situations. More precisely, we can give answers to the following two questions:

(Q1) Given a reference trajectory and initial conditions, can we ensure a priori that the closed-loop trajectories avoid the singularities?

(Q2) What can be done around the singularity, i.e. in the case of the unicycle robot, when the velocities become very low?

The following lemma provides sufficient conditions on the reference trajectory and on the initial conditions ensuring that the closed-loop trajectories are bounded away from the singularities, answering question (Q1).

Lemma 4.5 ([7], Chap. 8, [3]). Let system $\Sigma$ defined by (3.9), be generically fully linearizable by dynamic state feedback and diffeomorphim $\zeta=\Psi(\bar{X})$ given by (4.6). If:

(1) there exists an open simply connected domain $D$ included in

$$
S=\left\{\zeta \in \mathbb{R}^{\bar{n}} \text { such that } \Delta\left(\Psi^{-1}(\zeta)\right) \text { is not singular, } \Delta \text { being the decoupling matrix }\right\},
$$

(2) the reference trajectory $\zeta_{r}(t)$ belongs to $D$ for all $t$ and moreover there exists a positive continuous function $M_{1}(t)$ such that:

$$
\|\tilde{\zeta}(t)\|<M_{1}(t) \Rightarrow \zeta(t) \in D
$$

where $\tilde{\zeta}(t)=\zeta(t)-\zeta_{r}(t)$,

(3) the initial conditions satisfy:

$$
\|\tilde{\zeta}(0)\| \leq \min _{t} F(t) \text { with } F(t)=\frac{M_{1}(t)-\epsilon}{K_{2}(t)}
$$

for some positive constant $\epsilon$ and where $K_{2}(t)=K_{1} e^{-\lambda t}>0$ characterizes the closed-loop exponentially stable dynamics (with $K_{1}>0$ and $\lambda>0$ ):

$$
\|\tilde{\zeta}(t)\| \leq K_{2}(t)\|\tilde{\zeta}(0)\|, \forall t .
$$


Then:

$$
\zeta(t) \in D, \forall t
$$

Proof. To show (4.17), it is sufficient to show that:

$$
\|\tilde{\zeta}(t)\|<M_{1}(t), \forall t .
$$

By contradiction, let us suppose that there exists a positive time $T$ such that:

$$
\|\tilde{\zeta}(t)\|<M_{1}(t), \forall t \in\left[0, T\left[\text { and }\|\tilde{\zeta}(T)\|=M_{1}(T) .\right.\right.
$$

On $[0, T[$ we have from (4.15) and (4.16):

$$
\|\tilde{\zeta}(t)\|<M_{1}(t)-\epsilon
$$

and by continuity of $\tilde{\zeta}(t)$ and $M_{1}(t)$, equation (4.20) is in contradiction with (4.19) which concludes the proof.

Remark 4.6. It can be easily shown that the following choice of $M_{1}(t)$ satisfies (4.14):

$$
M_{1}(t)=\min _{\zeta}\|\tilde{\zeta}(t)\| \text { when } \operatorname{det}\left(\Delta\left(\Psi^{-1}(\zeta)\right)\right)=0 .
$$

The following lemma will now characterize the singularities and the function $M_{1}(t)$ of Lemma 4.5 for unicycle robots.

Lemma 4.7. For unicycle robots, we have:

$$
M_{1}(t)=\left|\left(\chi_{1}\right)_{r}(t)\right|,
$$

where $\left(\chi_{1}\right)_{r}(t)$ is the reference trajectory of the longitudinal velocity of point $G$ with coordinates $(x, y)^{\top}($ see Fig. 1). Consequently, if condition (4.15) is satisfied with (4.22), then the robot will follow the reference trajectory without singularity.

Proof. From (4.21), (4.12) and $\chi_{1}=v_{1}= \pm \sqrt{\dot{x}^{2}+\dot{y}^{2}}$, we easily obtain:

$$
M_{1}(t)=\min \left|\left(x-x_{r}\right)^{2}+\left(y-y_{r}\right)^{2}+\left(\dot{x}-\dot{x}_{r}\right)^{2}+\left(\dot{y}-\dot{y}_{r}\right)^{2}\right|^{\frac{1}{2}} \text { when } \chi_{1}=v_{1}=0,
$$

i.e.

$$
M_{1}(t)=\left|\left(\chi_{1}\right)_{r}(t)\right|
$$

Moreover, the diffeomorphism $\Psi(\bar{X})$ given by (4.12) is singular when $\chi_{1}=0$. Let us introduce:

$$
S^{+}=\left\{\bar{X} \in \mathbb{R} \times \mathbb{R} \times \frac{\mathbb{R}}{2 \pi \mathbb{Z}} \times \mathbb{R}^{*+}\right\},
$$

and similarly

$$
S^{-}=\left\{\bar{X} \in \mathbb{R} \times \mathbb{R} \times \frac{\mathbb{R}}{2 \pi \mathbb{Z}} \times \mathbb{R}^{\star-}\right\} .
$$

$\Psi$ can be inverted respectively on $\Psi\left(S^{+}\right)$and $\Psi\left(S^{-}\right)$as follows:

$$
\bar{X}=\Psi^{-1}(\zeta)=\left(x, y,-\arctan \left(\frac{\dot{x}}{\dot{y}}\right)+f(\dot{y}) \pi, \sqrt{\dot{x}^{2}+\dot{y}^{2}}\right)^{\top} \text { on } \Psi\left(S^{+}\right),
$$


and

$$
\bar{X}=\Psi^{-1}(\zeta)=\left(x, y,-\arctan \left(\frac{\dot{x}}{\dot{y}}\right)+(1-f(\dot{y})) \pi,-\sqrt{\dot{x}^{2}+\dot{y}^{2}}\right)^{\top} \text { on } \Psi\left(S^{-}\right),
$$

where:

$$
f(a)=\left\{\begin{array}{l}
0 \text { if } a \geq 0 \\
1 \text { if } a<0
\end{array}\right.
$$

and

$$
\arctan \left(\frac{a}{b}\right)=\operatorname{sign}(a) \frac{\pi}{2} \text { when } b=0 .
$$

Therefore, $\Psi^{-1}$ is well-defined as soon as the sign of $\chi_{1}(0)$ is defined. The conclusion then holds by applying Lemma 4.5.

Remark 4.8. For a reference trajectory not to be singular, $\left(\chi_{r}\right)(t)$ must always keep the same sign and the same holds for the actual closed-loop trajectory. Consequently, $\chi_{1}(0)$ and $\left(\chi_{1}\right)_{r}(0)$ must have the same sign. Provided condition (4.15) is satisfied, if $\left(\chi_{1}\right)_{r}(0)>0\left(\operatorname{resp} .\left(\chi_{1}\right)_{r}(0)<0\right)$ the robot will follow the reference trajectory with the free wheel backward (resp. forward).

\section{Simulation results on a unicycle robot:}

We have applied these results to the posture tracking problem of a unicycle robot, choosing the flat outputs, i.e. the coordinates $(x, y)^{\top}$ of point $G$ (see Fig. 1 ). The singularity corresponds to $\chi_{1}=v_{1}=0$, or $\eta_{1}=0$ using the notation of the general kinematic models $(2.1)$. We consider the following moving reference trajectory which is not singular, i.e. such that $\left(\chi_{1}\right)_{r}(t)=\left(\eta_{1}\right)_{r}(t) \neq 0$ for all $t \geq 0$ :

$$
x_{r}(t)=y_{r}(t)=\frac{\sqrt{2} \cdot 10^{-2}}{3}(10-2 t)^{3}-\frac{\sqrt{2}}{2} t+\frac{5 \sqrt{2}}{6},
$$

such that the point $G$ will follow a straight line, with initial conditions:

$$
\left\{\begin{array}{l}
x(0)=x_{r}(0)-0.54=5.35 \mathrm{~m} \\
y(0)=y_{r}(0)+0.54=6.43 \mathrm{~m} \\
\dot{x}(0)=\dot{y}(0)=(\dot{x})_{r}(0)=(\dot{y})_{r}(0)=-3.53 \mathrm{~m} / \mathrm{s} .
\end{array}\right.
$$

On the other hand, the second order linear dynamics of the closed-loop error in $\left(x(t)-x_{r}(t)\right)$ and $\left(y(t)-y_{r}(t)\right)$ have been chosen with two real eigenvalues equal to -1 . Therefore, by Lyapunov arguments, it is easy to show that a possible $K_{2}(t)$ function in Lemma 4.5 is the following:

$$
K_{2}(t)=2 \sqrt{2}\left(1+\frac{1}{\sqrt{2}} \exp \left(-\left(1-\frac{1}{\sqrt{2}}\right) t\right)\right) .
$$

Moreover, from (4.22) we obtain:

$$
M_{1}(t)=0.04(10-2 t)^{2}+1.0 .
$$

Consequently, with $\epsilon=10^{-2}$, it is easy to obtain the function $F(t)$ introduced in Lemma 4.5 (see Fig. 4) and to check that with our choice (4.24), condition (4.15) is satisfied, and no singularity will be encountered. The simulation results showing the reference trajectory and the actual robot trajectory in the cartesian plane are displayed in Figure 4 as well as the trajectory errors on $x, y$ and $\eta_{1}$.

\subsubsection{The slider:}

As for the unicycle robot, considering (2.6) shows that the input $\tau_{1}=\chi_{1}$ has to be delayed twice to make the second input $\tau_{2}$ appear in the fourth order time-derivatives of $x$ and $y$. Let us then introduce the added state $\chi_{1}=\tau_{1}$ and the new input $U_{1}$ (which is nothing but the second time-derivative of $\tau_{1}$ ):

$$
\ddot{\chi}_{1}=U_{1} \text {. }
$$


a)

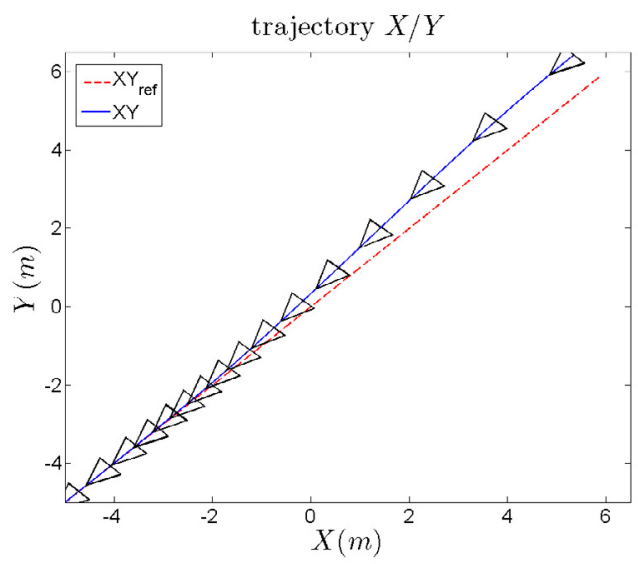

c)

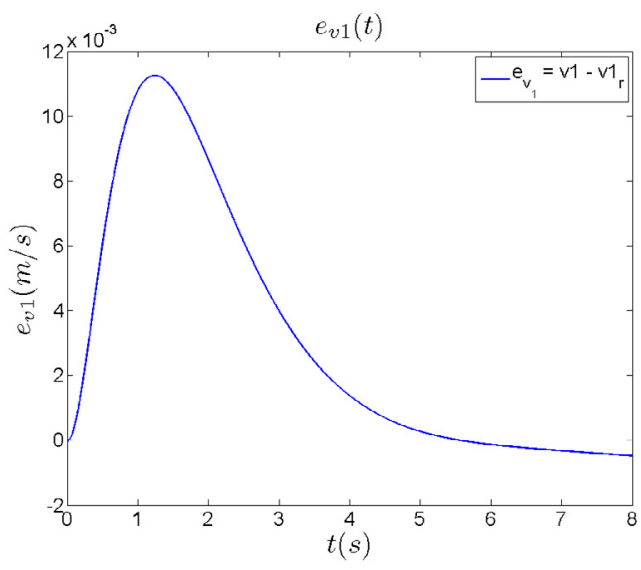

b)

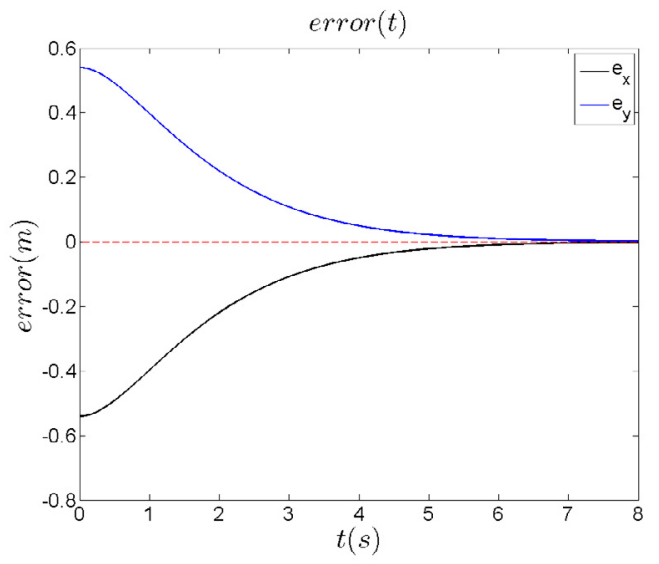

FiguRE 4. Tracking non singular reference trajectories for the unicycle robot using dynamic feedback linearization.

Then, computing $x^{(4)}$ and $y^{(4)}$, the extended system with extended vector $\bar{X}=\left(x, y, \dot{x}, \dot{y}, \psi, \dot{\psi}, \chi_{1}, \dot{\chi}_{1}\right)^{\top}$ is now fully linearizable by means of static state feedback with new input vector $\left(U_{1}, \tau_{2}\right)^{\top}$ and diffeomorphism:

$$
\Psi(\bar{X})=\left(x, y, \dot{x}, \dot{y}, \ddot{x}, \ddot{y}, x^{(3)}, y^{(3)}\right)^{\top} .
$$

More precisely, we have:

$$
\left(\begin{array}{c}
x^{(4)} \\
x^{(4)}
\end{array}\right)=\frac{1}{m}\left(\begin{array}{c}
-2 \dot{\chi}_{1} \dot{\psi} \sin \psi-\dot{\psi}^{2} \chi_{1} \cos \psi \\
2 \dot{\chi}_{1} \dot{\psi} \cos \psi-\dot{\psi}^{2} \chi_{1} \sin \psi
\end{array}\right)+\Delta\left(\begin{array}{c}
U_{1} \\
\tau_{2}
\end{array}\right) \text { with } \Delta=\frac{1}{m}\left(\begin{array}{c}
\cos \psi-\frac{\chi_{1} \sin \psi}{I} \\
\sin \psi \frac{\chi_{1} \cos \psi}{I}
\end{array}\right),
$$

and the new decoupling matrix $\Delta$ is generically invertible. The only singularity corresponds to $\operatorname{det}(\Delta)=0$ which is equivalent to $\chi_{1}=\tau_{1}= \pm m \sqrt{\ddot{x}^{2}+\ddot{y}^{2}}=0$. It means that the longitudinal acceleration of the slider cannot be zero, or in other words, that the robot's velocity must not remain constant.

As mentioned in Remark 4.3, let us check in the following proposition that $x$ and $y$ are flat outputs for the slider (2.6).

Proposition 4.9. The slider robot (2.6) is flat with outputs $Y_{1}=x$ and $Y_{2}=y$. 


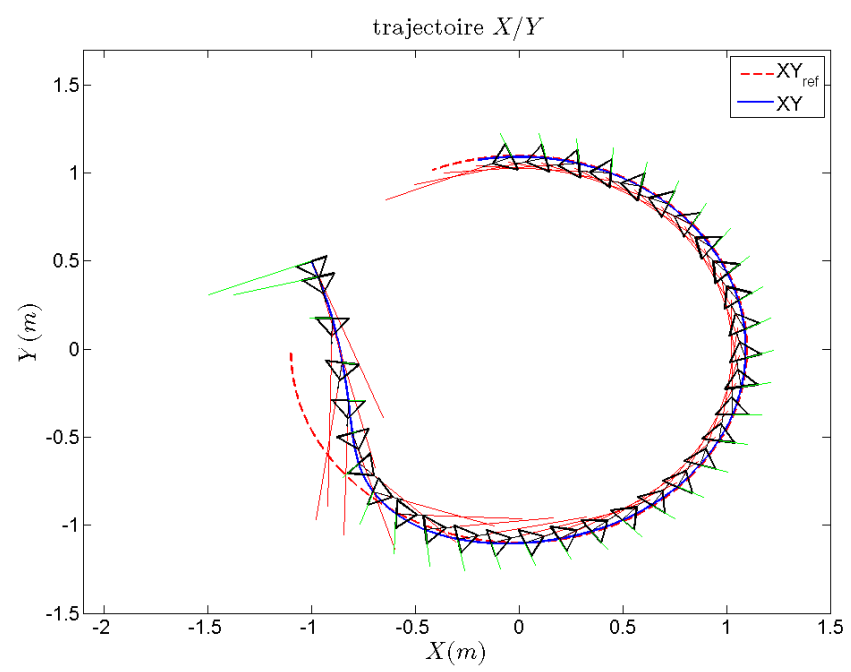

Figure 5. Posture tracking of a circle by a slider using dynamic linearizing feedback.

Proof. Using (2.6), it can be easily seen that:

$$
\psi=\arctan \left(\frac{\ddot{y}}{\ddot{x}}\right) \text { and } \tau_{1}= \pm m \sqrt{\ddot{x}^{2}+\ddot{y}^{2}} .
$$

It results that $\tau_{2}=\ddot{\psi}$ can be written as a function of $\left(\ddot{x}, \ddot{y}, x^{(3)}, y^{(3)}, x^{(4)}, y^{(4)}\right)^{\top}$, which means that $x$ and $y$ are flat outputs and concludes the proof.

Remark 4.10. In fact, to avoid too numerous time-derivatives of the outputs, we often consider $\dot{\psi}$ in $(2.6)$ as an input $v_{2}$, and then a backstepping approach can be handled to control the global cascaded system (2.6):

$$
\left\{\begin{array}{l}
m \ddot{x}=C_{\psi} \tau_{1} \\
m \ddot{y}=S_{\psi} \tau_{1} \\
\dot{\psi}=v_{2} .
\end{array}\right.
$$

We have applied this idea for simulation tests of posture tracking of a slider in the following simulations, as well as for experimental validation on a terrestrial quadrotor described below.

\section{Simulation results on a slider:}

In Figure 5, the slider follows a circular reference trajectory with radius $1.1 \mathrm{~m}$ centered at the origin, initial cartesian position $(-1.1,0)^{\top}$. The actual cartesian position of the slider is $(-0.8,0.5)^{\top}$ and its orientation is $\psi(t=0)=-1.2 \mathrm{rad}$. The red dotted line represents the circular reference trajectory and triangles with arrows represent the position and orientation of the slider at different regular time intervals. The actual longitudinal velocity (resp. lateral velocity) is represented by red lines (resp. green lines).

\section{Experimental results on a terrestrial quadrotor:}

A recent interest in aerial mobile robotics appears and more precisely for quadrotor platforms due to their mechanical simplicity and stability in flight. However, the energy consumption remains a major technological limitation. This is the reason why terrestrial quadrotors have already appeared on the market since they can move on the ground to save energy and fly to avoid obstacles. We have been interested in studying such a platform of an hybrid terrestrial and aerial quadrotor displayed in Figure 6. 


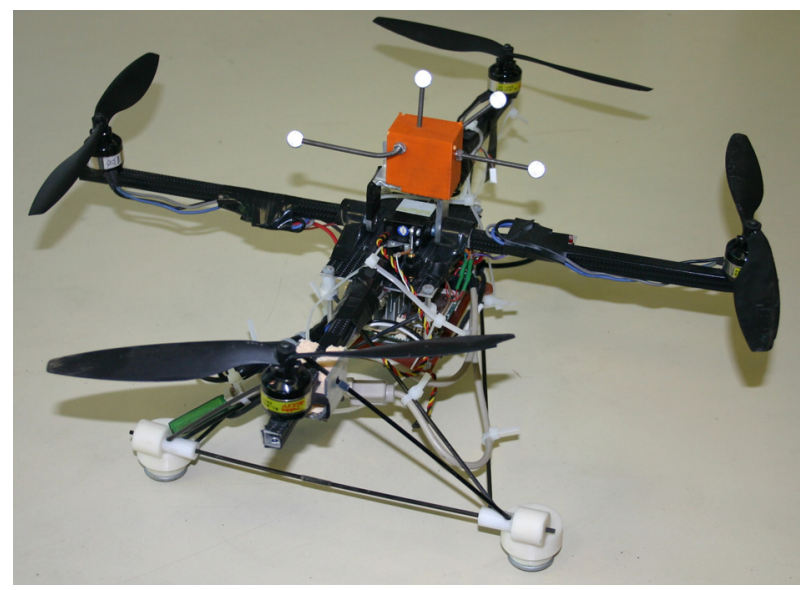

FiguRE 6 . The terrestrial quadrotor.

The base $B_{1}$ of the drone is supported on a moving platform $B_{0}$ where three omnidirectional ball casters are fixed and allow the drone to move freely in all directions, even sideways. Another body $B_{2}$ containing the four propellers is supported by the drone base $B_{1}$ through a revolute joint which can be directly actuated by a servo-motor which controls the pitch angle $\theta$ of $B_{2}$ (see [47-49] for a more detailed description).

In fact, the dynamical equations of this quadrotor are quite similar to the slider dynamics (4.30) since they can be written:

$$
\left\{\begin{array}{l}
\ddot{x}=\cos (\psi) p \\
\ddot{y}=\sin (\psi) p \\
\dot{\psi}=v_{2} \\
\dot{\theta}=v_{3},
\end{array}\right.
$$

where $p=\sin (\theta) u_{1}, \theta$ being the pitch angle. It can be pointed out that the longitudinal propulsion force $u_{1}=k \sum_{i=1}^{4} \omega_{i}^{2}$, where $k>0$ and $\omega_{i}$ denote the rotational velocities of the propellers, remains always positive so that the propellers cannot provide forces in the opposite direction. This can be realized using the pitch angle variable $\theta$ which is directly actuated. In fact, it can be easily shown that system (4.31) is flat using the flat outputs $(x, y, \theta)^{\top}$. The corresponding decoupling matrix is singular when the longitudinal acceleration $p$ is zero (as in the slider case) and when $\sin \theta=0$. Therefore, fisrt experimental results have been obtained for tracking non singular reference trajectories for our quadrotor through the flatness approach (see [47-49] for details).

More precisely, in order to test control laws in practice, a whole experimental system has been developed to produce the drone coordinates in real time for feedback control or data recording for diagnosis. A motion capture system with 6 Optitrack cameras placed around the tracking area allows us to track the position and orientation of the drone at $250 \mathrm{~Hz}$ with a sub-millimeter accuracy. We also obtain from the system the tilt angle of propellers owing to the orange part (see Fig. 6) where reflective markers are located. A remote computer linked to this Optitrack system gets back the position and orientation data; a software computes the control law algorithm and sends the controls to the drone via a ZigBee communication every $30 \mathrm{~ms}$. Figure 7 shows the experimental environment of our quadrotor.

We have applied a dynamic linearizing feedback law using the flat outputs to make the quadrotor track a circle at a mean longitudinal velocity of about $0.3 \mathrm{~m} / \mathrm{s}$. First experimental results are displayed in Figure 8 . The cartesian reference trajectory is plotted in red, the trajectory of the drone is the blue curve and the orientation of the drone is represented by triangles with arrows pointing in the longitudinal axis of the vehicle. During the circle tracking, the tilting angle $\theta$ is maintained to the constant value $\theta=0.8 \mathrm{rad}$. The control gains $\alpha_{i}^{j}$ (see (4.10) in Prop. 4.2) have been chosen equal to $\alpha_{x}^{0}=\alpha_{y}^{0}=0.21, \alpha_{x}^{1}=\alpha_{y}^{1}=2, \alpha_{x}^{2}=\alpha_{y}^{2}=0.26$. 


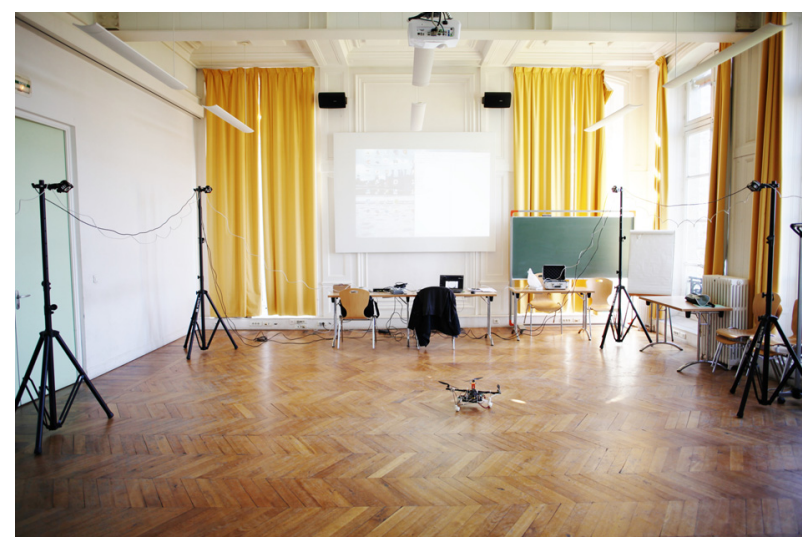

FiguRE 7. The experimental environment.

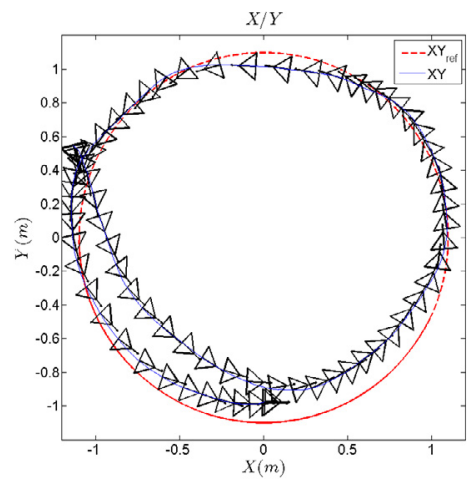

Figure 8. Experimental tracking of a circle by the terrestrial drone.

In Figure 9, some curves are displayed showing different state variables of the system during the circle tracking. Figures 9a, 9c, 9e and 9b, 9d, 9f represent respectively $x, \dot{x}, \ddot{x}$ and $y, \dot{y}, \ddot{y}$. The red curves represent the reference trajectories.

The result is quite satisfying despite noise disturbances and also friction effects which have been neglected in the control design which presents in fact a certain robustness. In addition to friction, we have chosen low gains in the control law in order to delay the slider's trajectory regarding to the reference one. This allows to avoid the singularity of the control law ensuring a strict positive acceleration. It must be pointed that the first and second derivatives of the measured positions $x$ and $y$ of the drone, obtained through the motion capture system, have been computed numerically and filtered. Nevertheless, due to important measurement noises, the acceleration signals remain noisy, but their average behavior follows quite well the corresponding reference signals.

Now we have seen how to proceed to avoid singularities when asymptotically tracking non singular reference trajectories, the question (Q2) remains, on what can be done when the linearizing control laws become singular, i.e. when the longitunal velocity (resp. acceleration) becomes too small for the unicycle robot (resp. the slider). Since the two systems are STLC, the idea is to elaborate time-varying (periodic) feedback laws to obtain asymptotic point stabilization for both systems (as shown in [11]), and to "switch" to those feedback laws when the linearizing control laws become too "high" (i.e. when the determinant of the decoupling matrix becomes too small). This kind of "hybrid" strategy switching to a stabilizing feedback law when the trajectory enters a sufficiently small disk centered at the origin has already been used for example in $[29,39,50]$. 


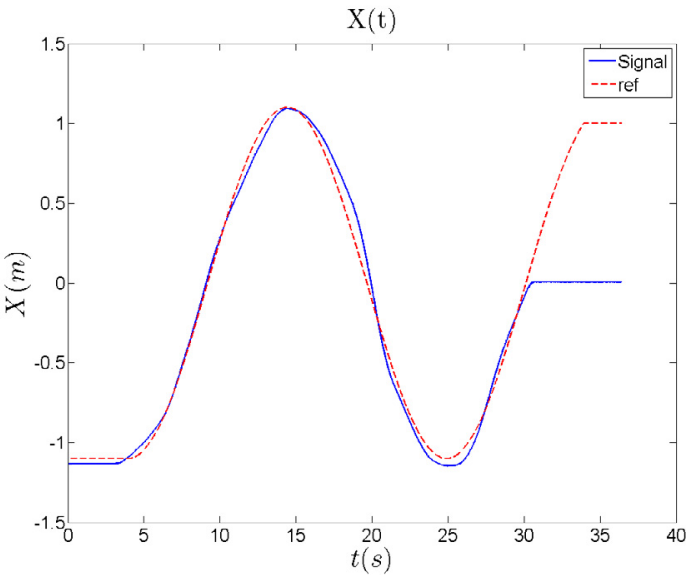

a)

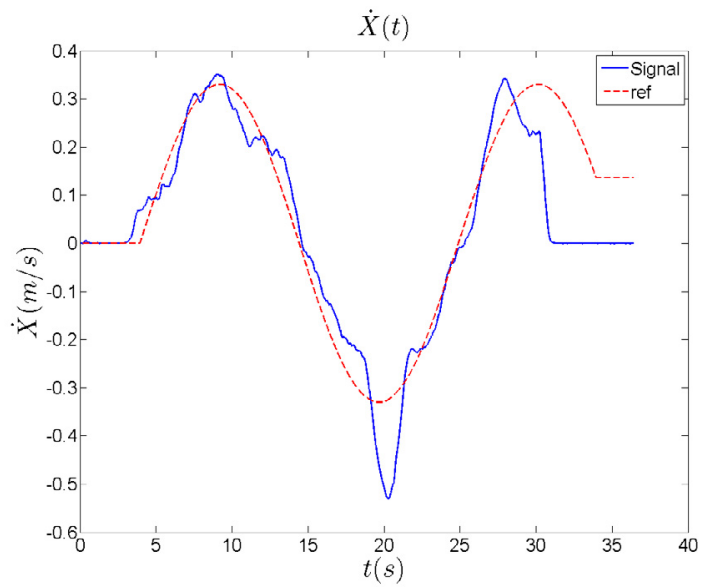

c)

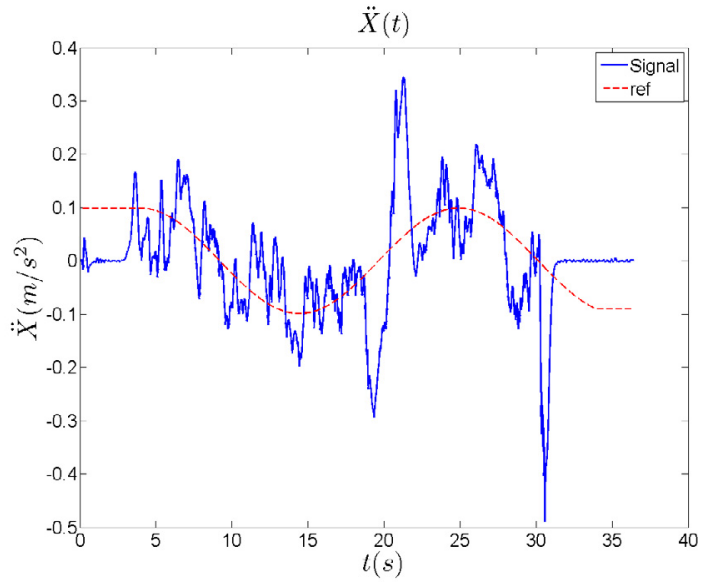

e)

Figure 9. Trajectories $x(t), \dot{x}(t), \ddot{x}(t)$ and $y(t), \dot{y}(t), \ddot{y}(t)$ of the drone. The corresponding reference trajectories are plotted in red. (Color online).

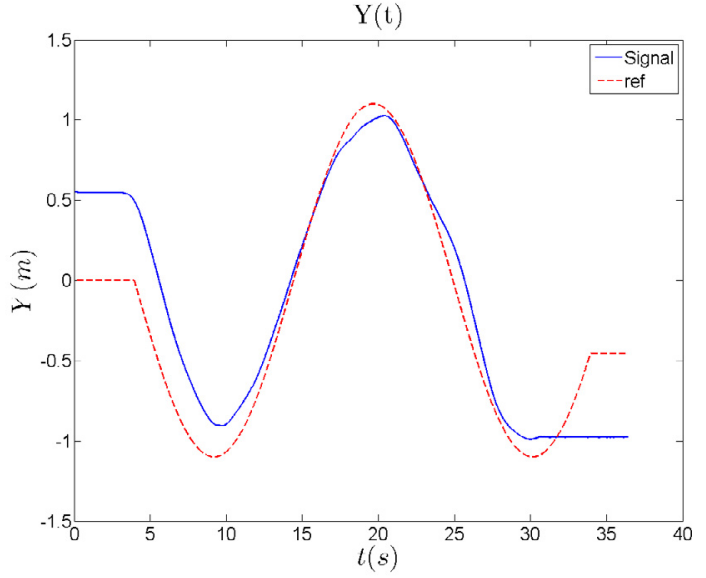

b)

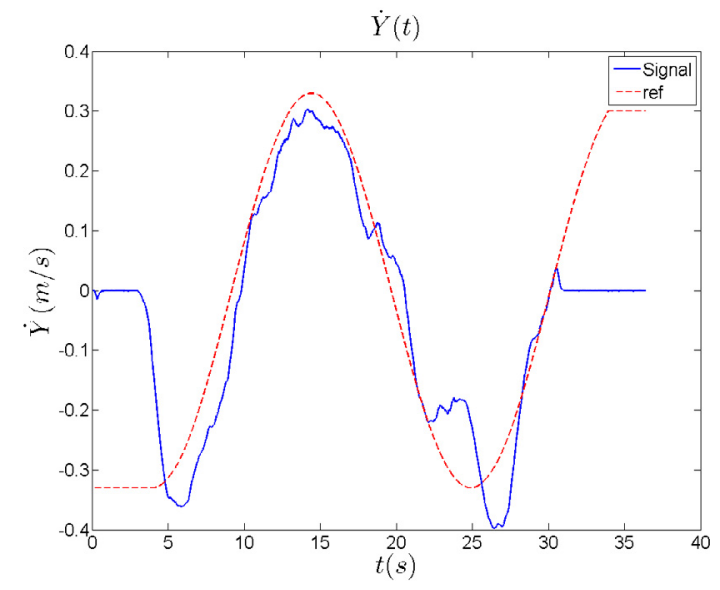

d)

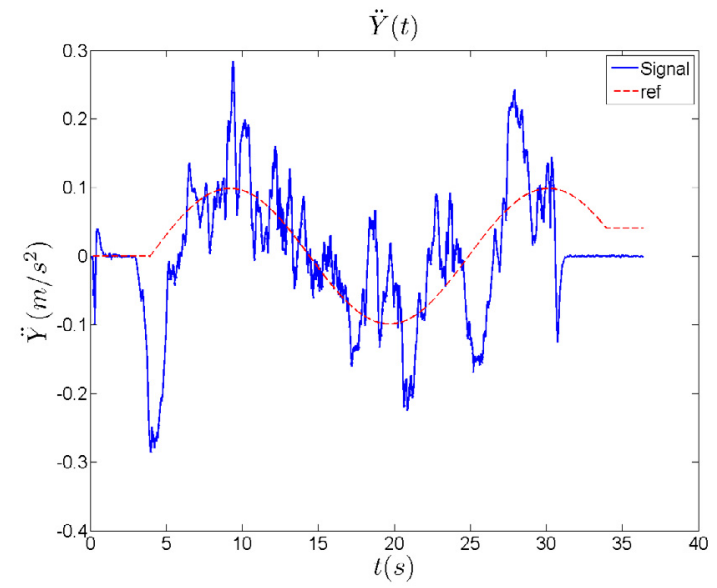

f)$$
\text { . }
$$ 


\subsection{Fixed point stabilization}

As we have seen in the previous section, state feedback laws, at least continuous, have to be time-varying to asymptotically stabilize fixed equilibrium points. For that purpose, we choose to elaborate such feedback control laws using the approach developed in [14] for dynamical systems of the form:

$$
\dot{X}=v f(X, u),
$$

where the state vector $X$ belongs to $\mathbb{R}^{n}, u \in \mathbb{R}^{m}$ and $v \in \mathbb{R}$ are the control inputs. This method has been successfully applied to equilibrium point stabilization of the unicycle robot (see [14]). We will extend the approach to the slider case.

Let us first recall the main stabilization results obtained in [14], in the particular case when $m=\operatorname{dim}(u)=1$.

Theorem 4.11 ([14]). Let us consider the following control inputs:

$$
\left\{\begin{array}{l}
u(X, t)=w(X) g(t) \\
v(X, t)=-q(X, u(X, t))
\end{array}\right.
$$

where:

(1)

$(2)$

(3)

$$
\begin{gathered}
g \in C^{\infty}(\mathbb{R},[-1 ; 1]) \\
\forall t \in \mathbb{R} \quad g(t+T)=g(t) \\
\forall t \in \mathbb{R}, \quad \exists l \in \mathbb{N}-\{0\} \quad g^{(l)}(t) \neq 0 \\
\text { for example, } \quad g(t)=\sin (\omega t), \\
w: \mathbb{R}^{n} \rightarrow \mathbb{R}, \quad w \in C^{\infty}\left(\mathbb{R}^{n}, \mathbb{R}\right) \\
\forall(0)=0 \\
\left\{\begin{array}{c}
\forall X \quad \text { s.t. } \quad w(X)=0 \Rightarrow q(X, 0) \neq 0 \\
\forall u \in[-|w(X)|,|w(X)|], \quad \quad \quad \frac{\partial^{r} q}{\partial u^{r}} \neq 0,
\end{array}\right.
\end{gathered}
$$

$$
\begin{aligned}
& q: \mathbb{R}^{n} \times \mathbb{R} \rightarrow \mathbb{R} \\
& q(X, u)=\sum_{i=1}^{n} \frac{\partial V}{\partial X_{i}}(x) f_{i}(X, u),
\end{aligned}
$$

where $V$ is a Lyapunov function candidate such that:

$$
\begin{gathered}
\quad V: \mathbb{R}^{n} \rightarrow \mathbb{R} \\
V(X) \rightarrow+\infty \quad \text { when }|X| \rightarrow+\infty, V(0)=0, \\
\forall X \in \mathbb{R}^{n}-\{0\} \quad V(X)>0 .
\end{gathered}
$$

If the control laws are chosen as in equation (4.33), then the origin $0 \in \mathbb{R}^{n}$ is globally asymptotically stable for the closed-loop system $(4.32) \dot{X}=v(X, t) f(X, u(X, t))$.

This result has been obtained in [14], considering the Lyapunov function $V(X)$ and LaSalle's invariance principle in the periodic case (see e.g. [20], Thm. 55.1). In fact, the result can be extended to the case $m \geq 2$ as shown in [14]. Let us now first apply this result to the fixed point stabilization of the unicycle robot.

\subsubsection{The unicycle robot}

We first consider the reduced system from the kinematic equations (2.4) of the unicycle robot:

$$
\left\{\begin{array}{l}
\dot{x}=v_{1} \cos (\psi) \\
\dot{y}=v_{1} \sin (\psi)
\end{array}\right.
$$


Theorem 4.11 applies with $X=(x, y)^{\top}$, the controls $v=v_{1}, u=\psi, f(X, u)=(\cos (\psi), \sin (\psi))^{\top}$ and $V(X)=$ $\frac{x^{2}+y^{2}}{2}$. Then,

$$
q(X, u)=x \cos (\psi)+y \sin (\psi), w(X)=y, g(t)=\sin (\omega t)
$$

satisfy assumptions of Theorem 4.11 and the following control laws:

$$
\left\{\begin{array}{l}
\psi=y \sin (\omega t) \\
v_{1}=-q(X, u(X, t))=-x \cos (y \sin (\omega t))-y \sin (y \sin (\omega t))
\end{array}\right.
$$

globally stabilize the origin of the reduced system (4.34). The time-derivative of the Lyapunov function $V$ is given by

$$
\dot{V}(X, t)=-(x \cos (\psi)+y \sin (\psi))^{2} \leq 0 .
$$

From LaSalle's invariance principle, it is necessary to excite the control variable $\psi$ as long as $y \neq 0$, to make the largest invariant subset included in $\{X$ s.t. $\dot{V}(X)=0\}$ equal to the origin $(x, y)=(0,0)$. This is realized by taking for example the suggested control $\psi=y \sin (\omega t)$ in equation (4.36).

To obtain now stabilizing control laws for the complete kinematic system (2.4), we have to consider the problem of cascaded systems using backstepping techniques (see e.g. [25, 40,46,51]). More precisely, a system obtained by adding pure integrators to a previous system which can be (globally) asymptotically stabilized by means of a smooth time-varying feedback law, can also be (globally) asymptotically stabilized by means of a smooth time-varying feedback law. This is a straightforward time-varying formulation of a result due to Tsinias [51]. More precisely, we recall the following lemma which has been proved in ([14], Lem. 1).

Lemma 4.12. Let us consider system $\Sigma$ given by:

$$
\begin{aligned}
& \dot{X}=F\left(X, u_{1}, u_{2}\right), X \in \mathbb{R}^{n}, \\
& u=\left(u_{1}, u_{2}\right)^{\top}: \mathbb{R}^{n} \times \mathbb{R} \rightarrow \mathbb{R}^{m_{1}} \times \mathbb{R}^{m_{2}},
\end{aligned}
$$

$F$ belonging to $C^{\infty}\left(\mathbb{R}^{n} \times \mathbb{R}^{m_{1}} \times \mathbb{R}^{m_{2}} ; \mathbb{R}^{n}\right)$. We make the following assumption on system (4.38):

A1: There exists a $C^{r} T$-periodic time-varying feedback law $u(X, t)$ such that $u(0, t)=0$ for all $t \in \mathbb{R}$, making 0 in $\mathbb{R}^{n}$ a globally asymptotically stable equilibrium point of $\dot{X}=F(X, u(X, t))$.

Under assumption $\mathbf{A} \mathbf{1}$, the following cascaded system $\bar{\Sigma}$

$$
\left\{\begin{array}{l}
\dot{X}=F\left(X, v_{1}, y\right), X \in \mathbb{R}^{n} \\
\dot{y}=v_{2}
\end{array}\right.
$$

where $y \in \mathbb{R}^{m_{2}}$, the control $v=\left(v_{1}, v_{2}\right)^{\top} \in \mathbb{R}^{m_{1}} \times \mathbb{R}^{m_{2}}$, is such that 0 in $\mathbb{R}^{n} \times \mathbb{R}^{m_{2}}$ is globally asymptotically stabilizable using a $C^{r-1} T$-periodic time-varying feedback law $v(X, y, t)$ such that $v(0,0, t)=0$ for all $t \in \mathbb{R}$.

Sketch of proof. From assumption A1, using a classical converse of Lyapunov's second theorem, we deduce that system (4.38) admits a $T$-periodic Lyapunov function $V(X, t)$. Let us consider the following Lyapunov function candidate for system $\bar{\Sigma}$ :

$$
\bar{V}(X, y, t)=\frac{1}{2}\left|y-u_{2}(X, t)\right|^{2}+V(X, t),
$$

and the following time-varying feedback law:

$$
\left\{\begin{array}{l}
v_{1}(X, y, t)=u_{1}(X, t), \\
v_{2}(X, y, t)=\frac{\partial u_{2}}{\partial t}(X, t)-\left(y-u_{2}(X, t)\right) \\
+\sum_{i=1}^{n} \frac{\partial u_{2}}{\partial X_{i}}(X, t) F_{i}\left(X, v_{1}(X, t), y\right)-H\left(X, u_{1}(X, t), u_{2}(X, t), y\right)\left(\frac{\partial V}{\partial X}(X, t)\right)^{\top},
\end{array}\right.
$$

where $H \in C^{\infty}\left(\mathbb{R}^{n} \times \mathbb{R}^{m_{1}} \times \mathbb{R}^{m_{2}} \times \mathbb{R}^{m_{2}} ; \mathcal{L}\left(\mathbb{R}^{m_{2}} ; \mathbb{R}^{n}\right)\right)$ satisfies:

$$
F\left(X, u_{1}, y\right)-F\left(X, u_{1}, u_{2}\right)=H^{\top}\left(X, u_{1}, u_{2}, y\right)\left(y-u_{2}\right),
$$


$\mathcal{L}\left(\mathbb{R}^{m_{2}} ; \mathbb{R}^{n}\right)$ denoting the set of linear maps from $\mathbb{R}^{m_{2}}$ into $\mathbb{R}^{n}$. Computing the time-derivative of $\bar{V}(X, y, t)$, it can be easily shown that the time-varying feedback law (4.41) makes $\bar{V}$ decrease and the conclusion holds. For details (see [14], Lem. 1).

Applying this result to system (2.4), the augmented following Lyapunov function can be considered:

$$
\bar{V}=V(x, y)+\frac{(\psi-y \sin (\omega t))^{2}}{2},
$$

with the following control laws:

$$
\left\{\begin{array}{l}
v_{1}=-K_{1}(x \cos (\psi)+y \sin (\psi)) \\
\Omega=\dot{y} \sin (\omega t)+\omega y \cos (\omega t)-K_{2}(\psi-y \sin (\omega t))
\end{array}\right.
$$

$K_{1}$ and $K_{2}$ being strictly positive constants, so that the time-derivative of $\bar{V}$ can be written:

$$
\dot{\bar{V}}(x, y, \psi, t)=-K_{1}(x \cos (\psi)+y \sin (\psi))^{2}-K_{2}(\psi-y \sin (\omega t))^{2} \leq 0 .
$$

Once more, the LaSalle's invariance principle allows to conclude to the asymptotic stabilization of the origin of the unicycle $(2.4)$, since $\{\dot{\bar{V}}=0\}$ implies that $\psi=y \sin (\omega t)$ and we are reduced to the previous case of system (4.34).

\section{Simulation results on a unicycle robot:}

The simulation experiment is displayed on Figure 10. The previous periodic control law (4.44) is implemented with $K_{1}=5, K_{2}=1$ and $\omega=3$. We can see on Figure 10 that this method is able to stabilize the unicycle robot to a fixed point $\left(x_{\text {ref }}, y_{\text {ref }}\right)$ with an imposed orientation angle $\psi_{\text {ref }}$.

Moreover, the simulation experiment corresponding to the hybrid control strategy is displayed on Figure 11. When $x^{2}+y^{2}=\frac{\sqrt{2}}{10}, \eta_{1}$ is sufficiently small and we have to switch to the time-periodic feedback law to obtain in a final step the stabilization of the robot at the origin (see also [39]). It can be seen on Figure 11 that $\eta_{1}$ is not zero before the commutation time.

\subsubsection{The slider}

We can apply the same approach to stabilize the velocity $\dot{x}, \dot{y}$ of the slider to the origin, and therefore to make the robot stop at a given cartesian position. As for the unicycle robot, we first consider the reduced system from the kinematic equations (2.6) of the slider:

$$
\left\{\begin{array}{l}
m \ddot{x}=\tau_{1} \cos (\psi) \\
m \ddot{y}=\tau_{1} \sin (\psi)
\end{array}\right.
$$

Theorem 4.11 applies with $X=(\dot{x}, \dot{y})^{\top}$, the controls $v=\frac{\tau_{1}}{m}, u=\psi, f(X, u)=(\cos (\psi), \sin (\psi))^{\top}$ and $V(X)=$ $\frac{\dot{x}^{2}+\dot{y}^{2}}{2}$. Then,

$$
q(X, u)=\dot{x} \cos (\psi)+\dot{y} \sin (\psi), w(X)=\dot{y}, g(t)=\sin (\omega t)
$$

satisfy assumptions of Theorem 4.11 and the following control laws:

$$
\left\{\begin{array}{l}
\psi=\dot{y} \sin (\omega t) \\
v=-q(X, u(X, t))=-\dot{x} \cos (\dot{y} \sin (\omega t))-\dot{y} \sin (\dot{y} \sin (\omega t))
\end{array}\right.
$$

globally stabilize the origin of the reduced system (4.46). The time-derivative of the Lyapunov function $V$ is given by

$$
\dot{V}(X, t)=-(\dot{x} \cos (\psi)+\dot{y} \sin (\psi))^{2} \leq 0 .
$$




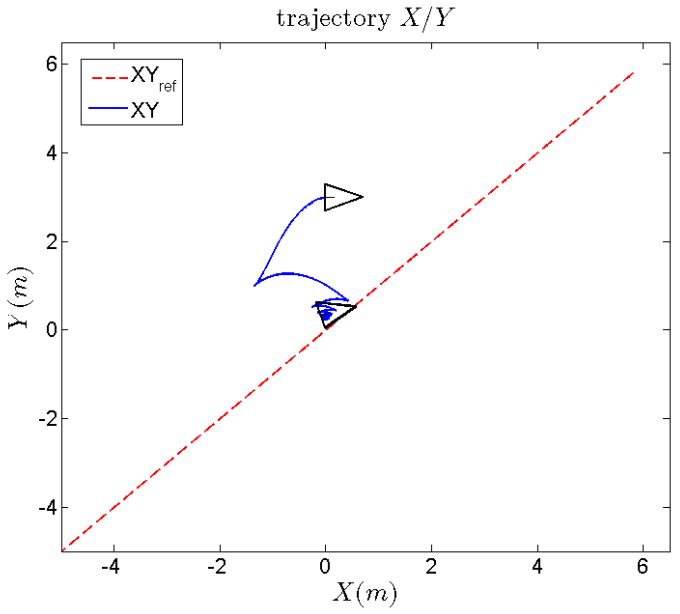

a)

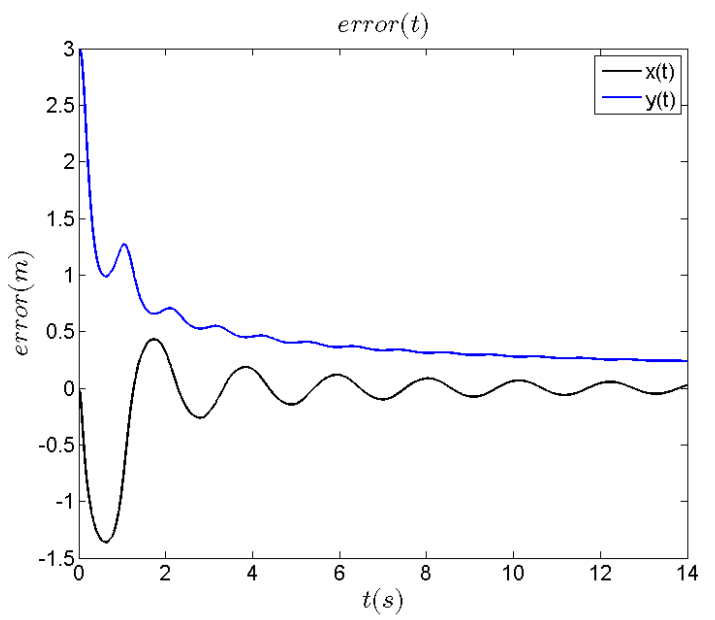

b)
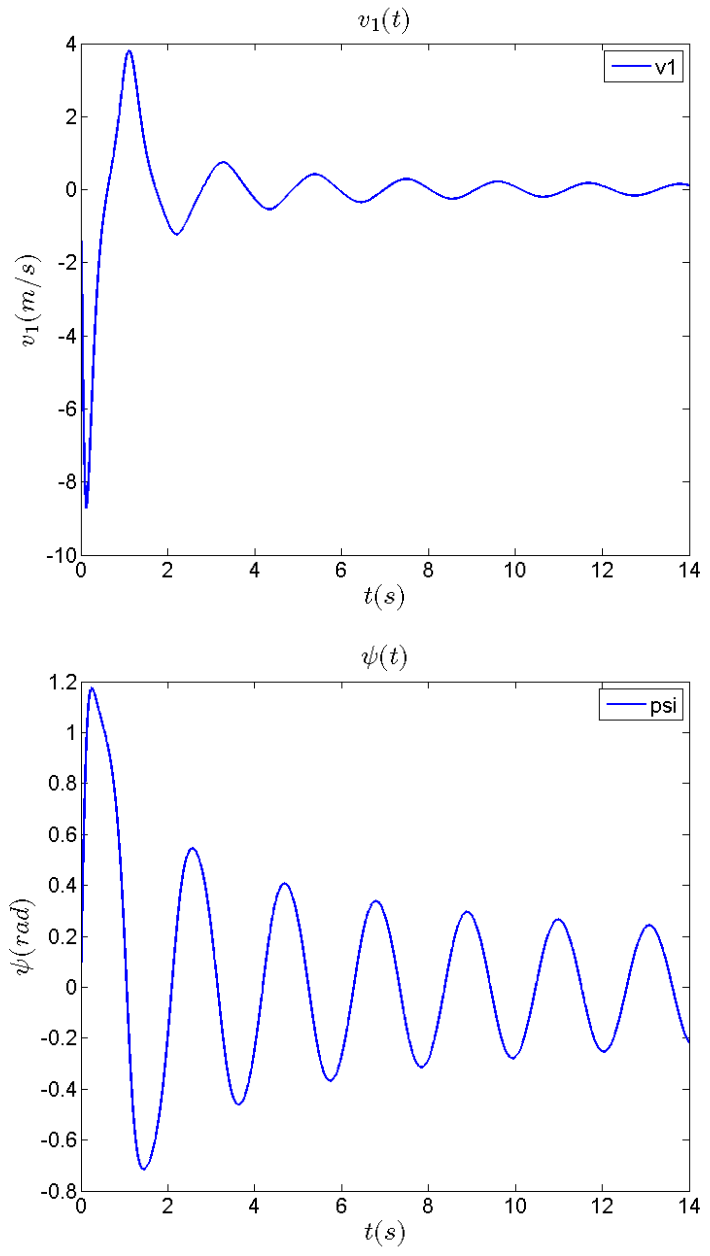

c)

FiguRE 10. Time-periodic feedback for unicycle stabilization at the rest point $x_{\mathrm{ref}}=0, y_{\mathrm{ref}}=0$ and $\psi_{\text {ref }}=0$. The initial state is $x_{0}=0, y_{0}=3, \psi=0$ and gains are $K_{1}=5, K_{2}=1$ and $\omega=3$.

From LaSalle's invariance principle, it is necessary to excite the control variable $\psi$ as long as $\dot{y} \neq 0$, to make the largest invariant subset included in $\{X$ s.t. $\dot{V}(X)=0\}$ equal to the origin $(\dot{x}, \dot{y})=(0,0)$. This is realized by taking for example the suggested control $\psi=\dot{y} \sin (\omega t)$ in equation (4.48).

In fact, the complete dynamical equations of the slider are given by (2.6) and we would have to apply a backstepping approach to define the physical control input $\tau_{2}$ controlling the angular acceleration $\ddot{\psi}$. But, as explained in Remark 4.10, we can simplify the control of the slider considering equations (4.30) where the angular velocity $\dot{\psi}$ is considered as the control variable $v_{2}$ so that the backstepping method has to be applied once, and similarly to the unicycle robot, we can consider the augmented following Lyapunov function:

$$
\bar{V}=V(\dot{x}, \dot{y})+\frac{(\psi-\dot{y} \sin (\omega t))^{2}}{2}
$$



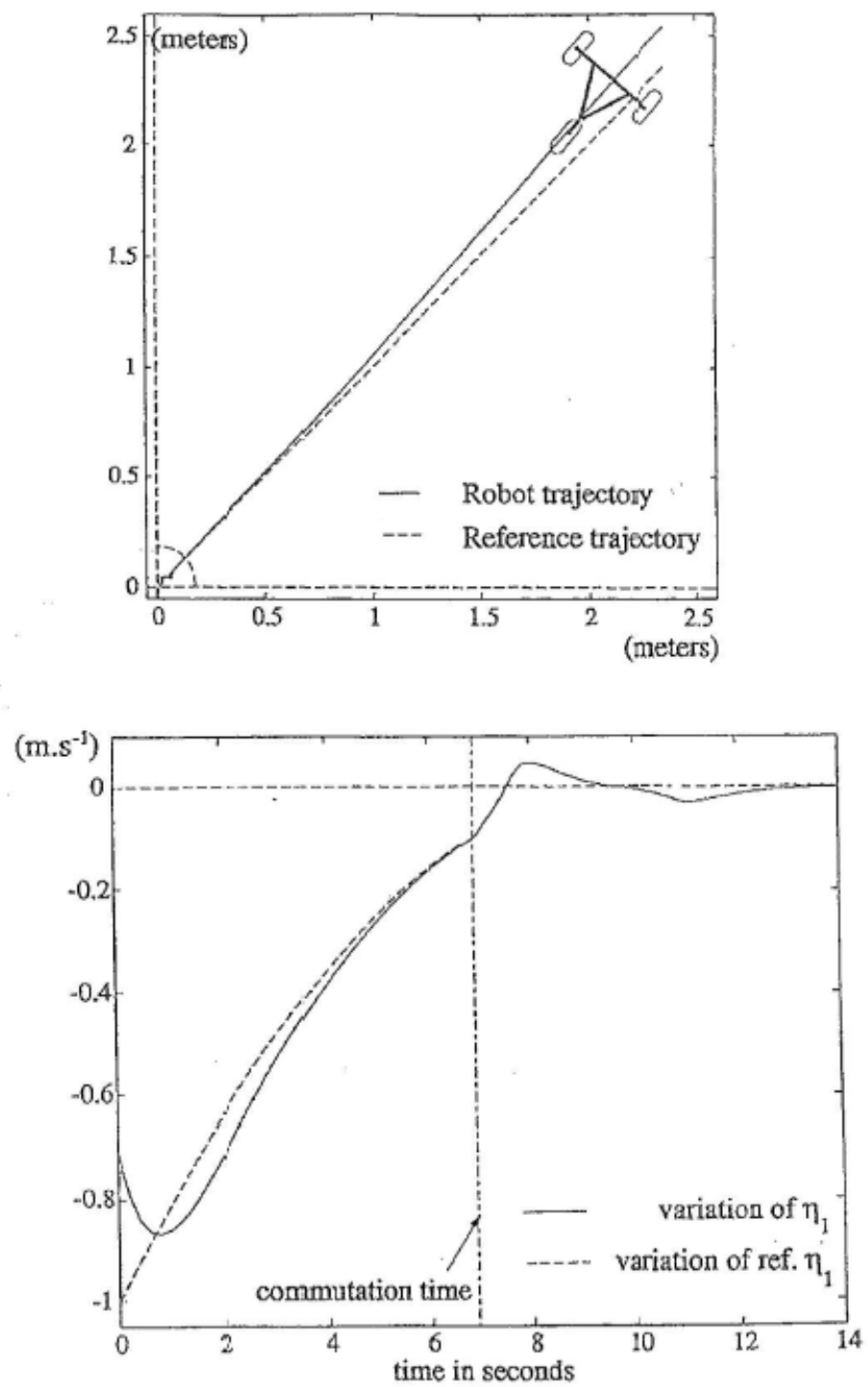

FIgURE 11. Tracking a singular reference trajectory for the unicycle robot using dynamic feedback linearization and time-periodic feedback for final stabilization at the rest point.

with the following control laws:

$$
\left\{\begin{array}{l}
v=-K_{1}(\dot{x} \cos (\psi)+\dot{y} \sin (\psi)) \\
v_{2}=\ddot{y} \sin (\omega t)+\omega \dot{y} \cos (\omega t)-K_{2}(\psi-\dot{y} \sin (\omega t))
\end{array}\right.
$$

$K_{1}$ and $K_{2}$ being strictly positive constants, so that the time-derivative of $\bar{V}$ can be written:

$$
\dot{\bar{V}}(\dot{x}, \dot{y}, \psi, t)=-K_{1}(\dot{x} \cos (\psi)+\dot{y} \sin (\psi))^{2}-K_{2}(\psi-\dot{y} \sin (\omega t))^{2} \leq 0 .
$$

Once more, the LaSalle's invariance principle allows to conclude to the asymptotic stabilization of the origin of (4.30), since $\{\dot{\bar{V}}=0\}$ implies that $\psi=\dot{y} \sin (\omega t)$ and we are reduced to the previous case of system (4.46). This control law has been successfully applied to stop the slider as shown in Figures 12 and 13 (see also [47, 48]). 


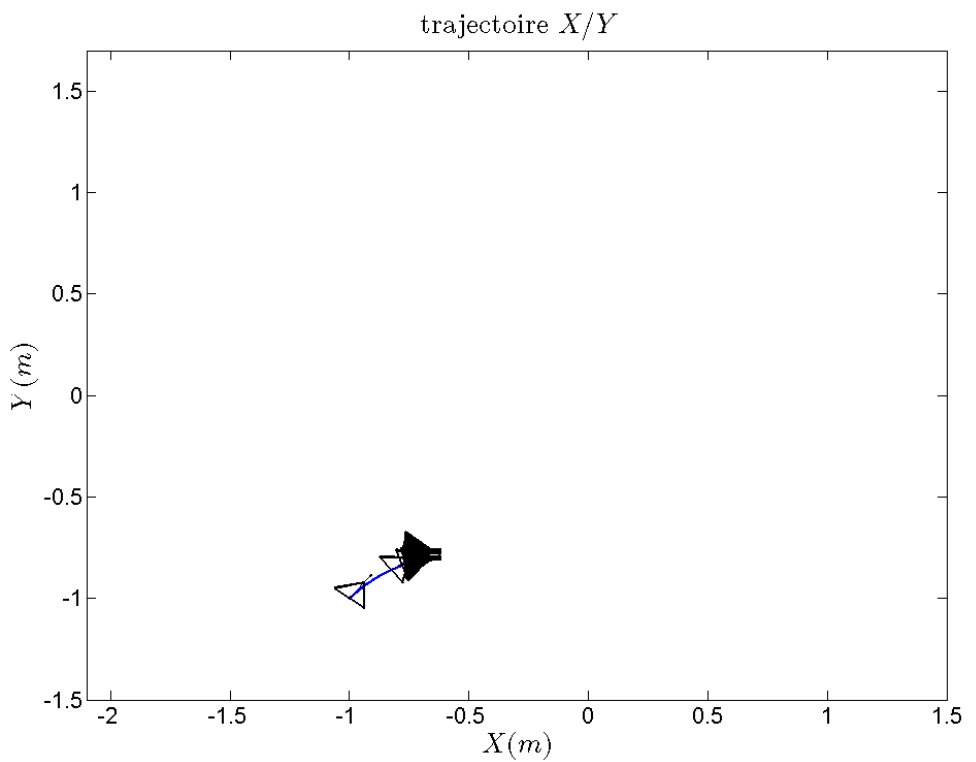

Figure 12. The slider in the cartesian plane, controlled with a time-varying periodic feedback law.

The Figure 12 shows the slider's cartesian trajectory, with initial conditions $x(0)=y(0)=-1 m, \dot{x}(0)=$ $\dot{y}(0)=0.6 \mathrm{~m} / \mathrm{s}, \ddot{x}(0)=0.3 \mathrm{~m} . \mathrm{s}^{-2}, \ddot{y}(0)=0.2 \mathrm{~m} . \mathrm{s}^{-2}$ and $\psi(0)=0.9 \mathrm{rad}$. The vehicle is represented by triangles with arrows representing the orientation of the slider at different regular time intervals. The time-varying control laws are known to provide low convergence rates, but we can increase the time response through the gains $K_{1}$ and $K_{2}$. In Figure 13, $x(t), y(t), \psi(t), \dot{x}(t)$ and $\dot{y}(t)$ are displayed, and it can be checked that, as expected, the vehicle stops $(\dot{x}(t)$ and $\dot{y}(t)$ converging to zero) with a zero final orientation.

Let us point out that this feedback law ensures the regulation to zero of the velocities $\dot{x}, \dot{y}$ and the orientation angle $\psi$ but if we want to regulate the position vector of the slider to a fixed chosen position, we have to elaborate another control design. To that purpose, we can consider the error tracking system (2.14) in the case of a reference fixed equilibrium point, that is with $\tau_{1 r}=\tau_{2 r}=0$. System can then be rewritten as follows:

$$
\left\{\begin{array}{l}
\Sigma_{1}:\left(\begin{array}{c}
\dot{e_{1}} \\
\dot{e_{2}} \\
\dot{e_{3}} \\
\dot{e_{4}}
\end{array}\right)=\left(\begin{array}{cccc}
0 & \dot{\psi} & 1 & 0 \\
-\dot{\psi} & 0 & 0 & 1 \\
0 & 0 & -\frac{d_{1}}{m} & \dot{\psi} \\
0 & 0 & -\dot{\psi} & -\frac{d_{2}}{m}
\end{array}\right)\left(\begin{array}{l}
e_{1} \\
e_{2} \\
e_{3} \\
e_{4}
\end{array}\right)+\left(\begin{array}{l}
0 \\
0 \\
1 \\
0
\end{array}\right) u_{1}, \\
\ddot{\psi}=-\frac{d_{3}}{I} \dot{\psi}+u_{2},
\end{array}\right.
$$

$\left(d_{3}, d_{2}, d_{1}>0\right.$ being the viscous friction coefficients (see (3.7)) with $u_{1}=\frac{\tau_{1}}{m}$ and $u_{2}=\frac{\tau_{2}}{I}$. Let $\Sigma_{1}$ be the sub-system of (4.53) described by the first four equations of system (4.53), i.e. by the dynamics of the $e_{i}$, $i=1, \ldots, 4 . \Sigma_{1}$ can be seen as a time-varying linear system, the state matrix $A(t)$ of which being time-varying through the evolution of the orientation angle $\psi(t)$. A first idea, inspired from [27], is to define $u_{1}$ ensuring the stabilization of the time-varying system $\Sigma_{1}$. The second step is then to use $u_{2}$, to make the yaw rate $\dot{\psi}$ persistently exciting when necessary.

\section{Stabilization of the sub-system $\Sigma_{1}$ :}

The following proposition gives a stabilizing control law $u_{1}(t)$ inspired from [27] making the origin of $\Sigma_{1}$ globally exponentially stable under some conditions on $\dot{\psi}(t)$. 

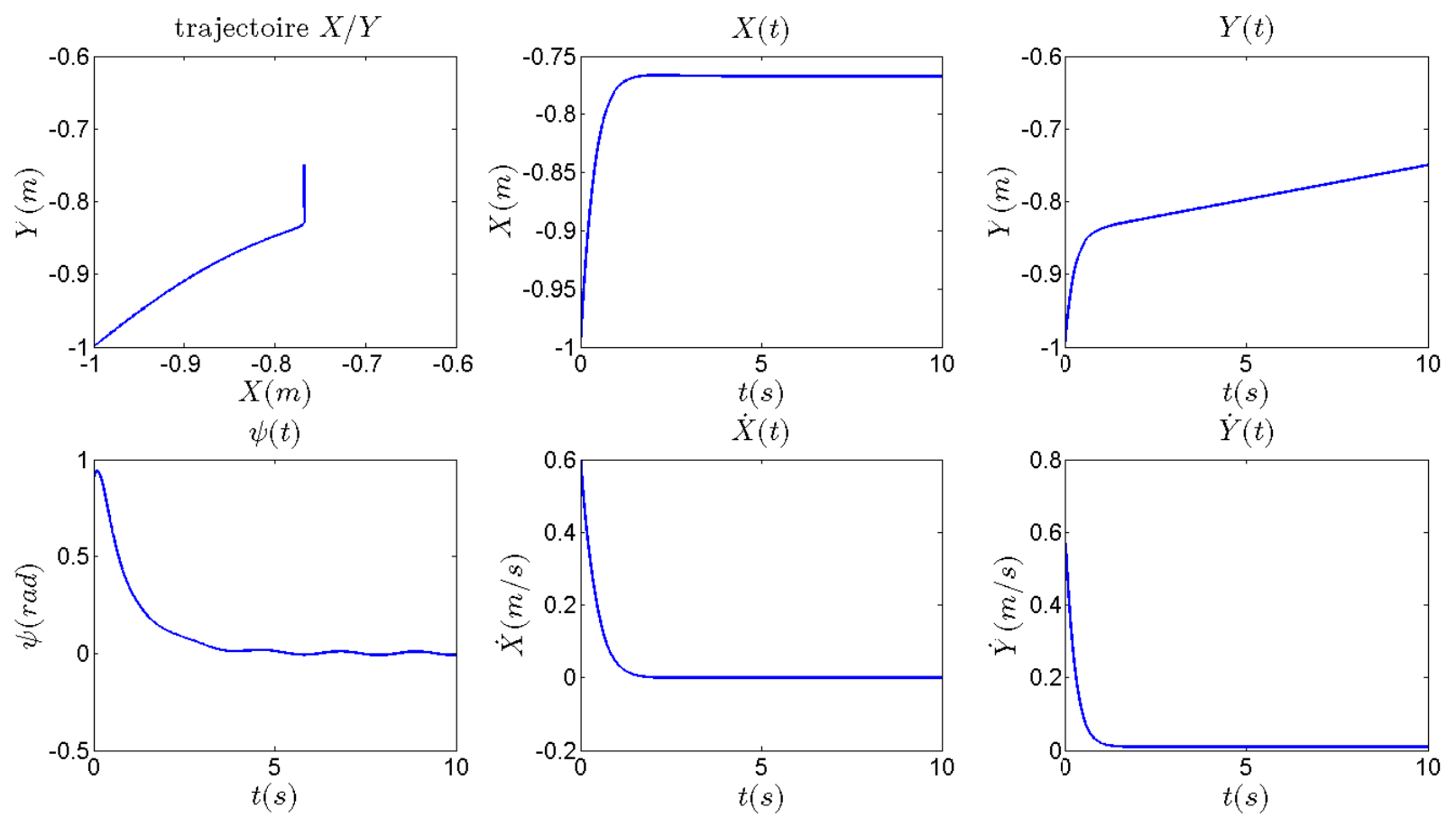

Figure 13. The cartesian trajectory, $x, y, \psi, \dot{x}$ and $\dot{y}$ of the slider.

Proposition 4.13. Let us consider $\Sigma_{1}$ in closed-loop with $u_{1}$ defined as follows:

$$
u_{1}(t)=\frac{1}{m}\left(-k_{3} e_{3}+k_{4} \dot{\psi}(t) e_{4}-k_{1} e_{1}+k_{2} \dot{\psi}(t) e_{2}\right)
$$

where the control gains $k_{i}$ satisfy the following inequalities:

$$
\begin{gathered}
k_{3}>d_{2}-d_{1}, \\
k_{4}=\frac{m k_{2}\left(k_{2}+k_{3}+d_{1}-d_{2}\right)}{d_{2} k_{2}+m k_{1}}, \\
0<k_{1}<\left(k_{3}+d_{1}-d_{2}\right) \frac{d_{2}}{m} \\
k_{2}>0
\end{gathered}
$$

If $\dot{\psi}(t)$ satisfies the assumptions of Proposition 3.11, then system $\Sigma_{1}$ in closed-loop with (4.54) is globally exponentially stable.

Proof. Let us suppose that the above inequalities (4.55) are satisfied and let us define $\lambda$ and $\mu(\lambda<\mu)$ as follows:

$$
\begin{gathered}
\lambda+\mu=\frac{k_{3}+d_{1}}{m}, \\
\lambda \mu=\frac{k_{1}}{m},
\end{gathered}
$$

$\lambda$ and $\mu$ are then roots of the polynomial $P(x)$ defined by:

$$
P(x)=m x^{2}-\left(k_{3}+d_{1}\right) x+k_{1} .
$$

The first inequality of (4.55) allows to write:

$$
0<\frac{d_{2}}{m}<\frac{k_{3}+d_{1}}{m} .
$$


Moreover,

$$
P(0)=P\left(\frac{k_{3}+d_{1}}{m}\right)=k_{1}>0,
$$

and

$$
P\left(\frac{d_{2}}{m}\right)=m\left(\frac{d_{2}}{m}\right)^{2}-\left(k_{3}+d_{1}\right) \frac{d_{2}}{m}+k_{1}=\left(d_{2}-k_{3}-d_{1}\right) \frac{d_{2}}{m}+k_{1}<0 .
$$

The intermediate value theorem shows that the roots $\lambda$ and $\mu$ are such that:

- $0<\lambda<\frac{d_{2}}{m}$ and $P(\lambda)=0$;

- $\frac{d_{2}}{m}<\mu<\frac{k_{3}+d_{1}}{m}$ and $P(\mu)=0$;

- $0<\lambda<\frac{d_{2}}{m}<\mu$.

These properties are useful to show the following inequalities

$$
\begin{gathered}
0<\mu-\lambda, \\
0<d_{2}-m \lambda, \\
0<m \mu-d_{2}, \\
0<m^{2} \lambda \mu+d_{2} k_{2}, \\
0<k_{2}+m \lambda, \\
0<k_{2}+m \mu .
\end{gathered}
$$

System $\Sigma_{1}$ in closed-loop with $u_{1}(t)$ given at Proposition 4.13, can then be written:

$$
\left(\begin{array}{c}
\dot{e_{1}} \\
\dot{e_{2}} \\
\dot{\dot{e}_{3}} \\
\dot{e_{4}}
\end{array}\right)=\left(\begin{array}{cccc}
0 & \dot{\psi}(t) & 1 & 0 \\
-\dot{\psi}(t) & 0 & 0 & 1 \\
-\frac{k_{1}}{m} & \frac{k_{2}}{m} \dot{\psi}(t) & -\frac{k_{3}+d_{1}}{m} & \left(\frac{k_{4}}{m}+1\right) \dot{\psi}(t) \\
0 & 0 & -\dot{\psi}(t) & -\frac{d_{2}}{m}
\end{array}\right)\left(\begin{array}{c}
e_{1} \\
e_{2} \\
e_{3} \\
e_{4}
\end{array}\right)
$$

This equation can be rewritten:

$$
\left(\begin{array}{c}
\dot{e_{1}} \\
\dot{e_{2}} \\
\dot{e_{3}} \\
\dot{e_{4}}
\end{array}\right)=\left(\begin{array}{cccc}
0 & \dot{\psi}(t) & 1 & 0 \\
-\dot{\psi}(t) & 0 & 0 & 1 \\
-\lambda \mu & \frac{k_{2}}{m} \dot{\psi}(t) & -(\lambda+\mu) & \frac{\left(k_{2}+m \lambda\right)\left(k_{2}+m \mu\right)}{m^{2} \lambda \mu+d_{2} k_{2}} \dot{\psi}(t) \\
0 & 0 & -\dot{\psi}(t) & -\frac{d_{2}}{m}
\end{array}\right)\left(\begin{array}{l}
e_{1} \\
e_{2} \\
e_{3} \\
e_{4}
\end{array}\right) .
$$

Let us now introduce a change of coordinates and let us denote $\left(x_{1}, x_{2}, x_{3}, x_{4}\right)^{\top}$ the new state vector, linked to $e$ by the following transformations (4.62) and (4.63):

$$
\left(\begin{array}{l}
e_{1} \\
e_{2} \\
e_{3} \\
e_{4}
\end{array}\right)=\left(\begin{array}{cccc}
0 & 0 & \frac{-1}{\mu-\lambda} & \frac{1}{\mu-\lambda} \\
\frac{m}{d_{2}} & -\frac{m}{d_{2}} & 0 & 0 \\
0 & 0 & \frac{\mu}{\mu-\lambda} & \frac{-\lambda}{\mu-\lambda} \\
0 & 1 & 0 & 0
\end{array}\right)\left(\begin{array}{l}
x_{1} \\
x_{2} \\
x_{3} \\
x_{4}
\end{array}\right)
$$




$$
\left(\begin{array}{l}
x_{1} \\
x_{2} \\
x_{3} \\
x_{4}
\end{array}\right)=\left(\begin{array}{cccc}
0 & \frac{d_{2}}{m} & 0 & 1 \\
0 & 0 & 0 & 1 \\
\lambda & 0 & 1 & 0 \\
\mu & 0 & 1 & 0
\end{array}\right)\left(\begin{array}{l}
e_{1} \\
e_{2} \\
e_{3} \\
e_{4}
\end{array}\right) .
$$

Let us point out that from the gains'choice (4.59) and by construction of the roots we have $\mu-\lambda>0$. The new system has then the following form:

$$
\left(\begin{array}{c}
\dot{x_{1}} \\
\dot{x_{2}} \\
\dot{x_{3}} \\
\dot{x_{4}}
\end{array}\right)=A_{1}(t)\left(\begin{array}{c}
x_{1} \\
x_{2} \\
x_{3} \\
x_{4}
\end{array}\right)
$$

with

$$
A_{1}(t)=\left(\begin{array}{cccc}
0 & 0 & -\frac{m \mu-d_{2}}{m(\mu-\lambda)} \dot{\psi}(t) & -\frac{d_{2}-m \lambda}{m(\mu-\lambda)} \dot{\psi}(t) \\
0 & -\frac{d_{2}}{m} & -\frac{\mu}{(\mu-\lambda)} \dot{\psi}(t) & \frac{\lambda}{\mu-\lambda} \dot{\psi}(t) \\
\frac{k_{2}+m \lambda}{d_{2}} \dot{\psi}(t) & \frac{m \mu\left(k_{2}+m \lambda\right)\left(d_{2}-m \lambda\right)}{d_{2}\left(m^{2} \lambda \mu+d_{2} k_{2}\right)} \dot{\psi}(t) & -\mu & 0 \\
\frac{k_{2}+m \mu}{d_{2}} \dot{\psi}(t) & -\frac{m \lambda\left(k_{2}+m \mu\right)\left(m \mu-d_{2}\right)}{d_{2}\left(m^{2} \lambda \mu+d_{2} k_{2}\right)} \dot{\psi}(t) & 0 & -\lambda
\end{array}\right) .
$$

The inequalities (4.59) allow to consider the following Lyapunov function candidate $V$ :

$$
V\left(x_{1}, x_{2}, x_{3}, x_{4}\right)=\frac{m}{2 d_{2}} x_{1}^{2}+\frac{m\left(d_{2}-m \lambda\right)\left(m \mu-d_{2}\right)}{2 d_{2}\left(m^{2} \lambda \mu+d_{2} k_{2}\right)} x_{2}^{2}+\frac{\left(m \mu-d_{2}\right)}{2\left(k_{2}+m \lambda\right)(\mu-\lambda)} x_{3}^{2}+\frac{\left(d_{2}-m \lambda\right)}{2\left(k_{2}+m \mu\right)(\mu-\lambda)} x_{4}^{2} .
$$

Computing the time-derivative of $V$ along the solutions of the dynamical system (4.64), we obtain:

$$
\dot{V}\left(x_{1}, x_{2}, x_{3}, x_{4}\right)=-\frac{\left(d_{2}-m \lambda\right)\left(m \mu-d_{2}\right)}{\left(m^{2} \lambda \mu+d_{2} k_{2}\right)} x_{2}^{2}-\frac{\mu\left(m \mu-d_{2}\right)}{\left(k_{2}+m \lambda\right)(\mu-\lambda)} x_{3}^{2}-\frac{\lambda\left(d_{2}-m \lambda\right)}{\left(k_{2}+m \mu\right)(\mu-\lambda)} x_{4}^{2} .
$$

Let us now introduce the matrix $C$ such that:

$$
C=\left(0 \sqrt{\frac{\left(m \mu-d_{2}\right)\left(d_{2}-m \lambda\right)}{m^{2} \lambda \mu+d_{2} k_{2}}} \sqrt{\frac{\mu\left(m \mu-d_{2}\right)}{(\mu-\lambda)\left(k_{2}+m \lambda\right)}} \sqrt{\frac{\lambda\left(d_{2}-m \lambda\right)}{(\mu-\lambda)\left(k_{2}+m \mu\right)}}\right),
$$

then we can write

$$
\dot{V}=-x^{\top} C^{\top}(t) C(t) x \leq 0 .
$$

The origin of system (4.64) is globally exponentially stable if the pair $\left(A_{1}(\dot{\psi}(t)), C\right)$ is uniformly completely observable (cf. [23], Chaps. 3, 3.4, 3.5). From Proposition 3.11 and by duality arguments, the pair $\left(A_{1}^{\top}(\dot{\psi}(t)), C^{\top}\right)$ is uniformly completely controllable if the pair $\left(A_{1}^{\top}(\dot{\psi}(t)), C^{\top}\right)$ satisfies the Kalman controllability condition if $\dot{\psi}(t) \neq 0$ (this rank condition can be easily checked see e.g. [47], Chap. 2) and if $\dot{\psi}(t)$ satisfies the assumptions (i), (ii) and (iii) of Proposition 3.11, which ends the proof. 


\section{Stabilization of the complete system (4.53):}

The idea is now to find the control law $u_{2}(t)$ making the yaw rate $\dot{\psi}(t)$ satisfying the assumptions of Proposition 3.11 and in addition making the orientation angle $\psi$ tend to a desired orientation $\psi_{r}$. In a first step, from Remark 4.10 and using backstepping arguments, we can consider $\dot{\psi}$ as the new control input. Let us introduce the following Lyapunov function candidate:

$$
\bar{V}=V+\frac{1}{2}\left(\psi-\psi_{r}+K e_{2} \sin (\omega t)\right)^{2},
$$

where $V$ is given by (4.66). We can prove the stabilization result for the slider in the following proposition.

Proposition 4.14. The control law $u_{1}$ given by (4.54) and $\dot{\psi}$ given by (4.71) solve the fixed point stabilization problem for the slider (4.53).

Proof. Computing the time-derivative of $\bar{V}$ gives:

$$
\begin{aligned}
\dot{\bar{V}}= & -\frac{\left(d_{2}-M \lambda\right)\left(M \mu-d_{2}\right)}{\left(M^{2} \lambda \mu+d_{2} k_{2}\right)} x_{2}^{2}-\frac{\mu\left(M \mu-d_{2}\right)}{\left(k_{2}+M \lambda\right)(\mu-\lambda)} x_{3}^{2}-\frac{\lambda\left(d_{2}-M \lambda\right)}{\left(k_{2}+M \mu\right)(\mu-\lambda)} x_{4}^{2} \\
& +\left(\left(1-K e_{1} \sin (\omega t)\right) \dot{\psi}-\omega K e_{2} \cos (\omega t)+K e_{4} \sin (\omega t)\right)\left(\psi-\psi_{r}+K e_{2} \sin (\omega t)\right) .
\end{aligned}
$$

Then, the following control input:

$$
\dot{\psi}=\frac{-K_{1}\left(\psi-\psi_{r}+K e_{2} \sin (\omega t)\right)-K e_{4} \sin (\omega t)+K \omega e_{2} \cos (\omega t)}{1-K e_{1} \sin (\omega t)},
$$

with $1-K e_{1} \sin (\omega t)>0$, produces:

$$
\begin{aligned}
\dot{\bar{V}}= & -\frac{\left(d_{2}-M \lambda\right)\left(M \mu-d_{2}\right)}{\left(M^{2} \lambda \mu+d_{2} k_{2}\right)} x_{2}^{2}-\frac{\mu\left(M \mu-d_{2}\right)}{\left(k_{2}+M \lambda\right)(\mu-\lambda)} x_{3}^{2}-\frac{\lambda\left(d_{2}-M \lambda\right)}{\left(k_{2}+M \mu\right)(\mu-\lambda)} x_{4}^{2} \\
& -K_{1}\left(\psi-\psi_{r}+K e_{2} \sin (\omega t)\right)^{2} .
\end{aligned}
$$

( $K$ should be chosen sufficiently small to avoid the singularity $1-K e_{1} \sin (\omega t)=0$ (see [47], Chap. 4, Sect. 4.5.6) for details). It is clear that $\dot{\bar{V}} \leq 0$. We can now apply LaSalle arguments for time-varying periodic systems. More precisely, the trajectories of the closed-loop system converge to the largest invariant sub-system $\mathcal{I}$ included in $\{\dot{\bar{V}}=0\}$.

$$
\mathcal{I} \subseteq\{\dot{\bar{V}}=0\}=\left\{x_{2}=0, x_{3}=0, x_{4}=0, \psi=\psi_{r}-K e_{2} \sin (\omega t)\right\} .
$$

Nevertheless, from (4.64) and (4.65), since $x_{3}=x_{4}=0$, we can deduce that $\dot{x}_{1}=0$. Therefore, from (4.62) and since $x_{2}=0$, we have $e_{2}=\frac{m}{d_{2}} x_{1}$, and then $\dot{e}_{2}=0$. Consequently, $e_{2}$ remains constant in $\mathcal{I}$ which implies that the control $\dot{\psi}$ in $\mathcal{I}$ has the following expression:

$$
\dot{\psi}=-K \omega e_{2} \cos (\omega t)
$$

where $e_{2}$ is constant. To conclude, if $\omega$ is chosen sufficiently small, $\dot{\psi}$ satisfies all the assumptions of Proposition 3.11 in $\mathcal{I}$ and, from Proposition 4.13, $x=0$ is globally exponentially stable which implies that $e_{2}$ tends to zero. Consequently, $\dot{\psi}$ tends to zero and $\psi$ tends to the desired reference value $\psi_{r}$, which concludes the fact that the periodic time-varying feedback law (4.71) ensures the fixed point stabilization problem. The stabilization of the whole slider's dynamics (4.53) can then be achieved through backstepping techniques (see Rem. 4.15).

This periodic control law was tested in simulation to stabilize the slider system to a fixed point, as displayed in Figures 14 and 15. More simulation results are available and detailed in [47]. 
a)

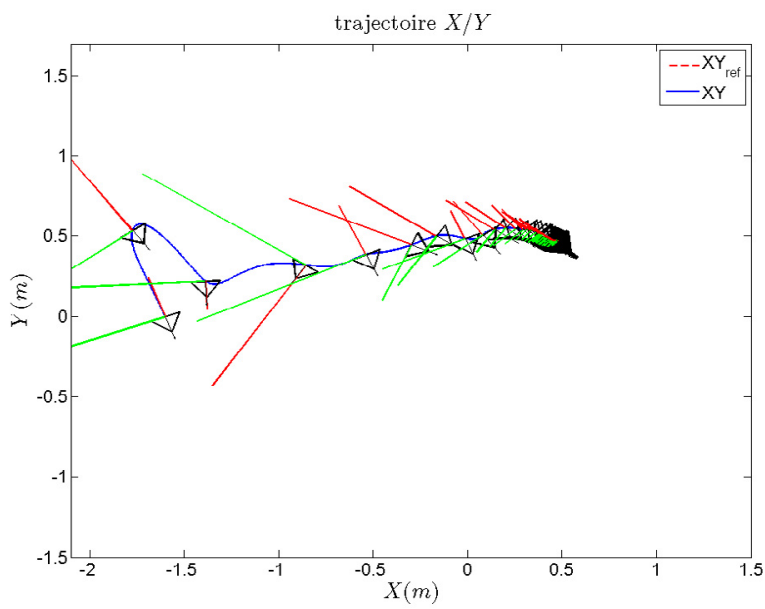

b)

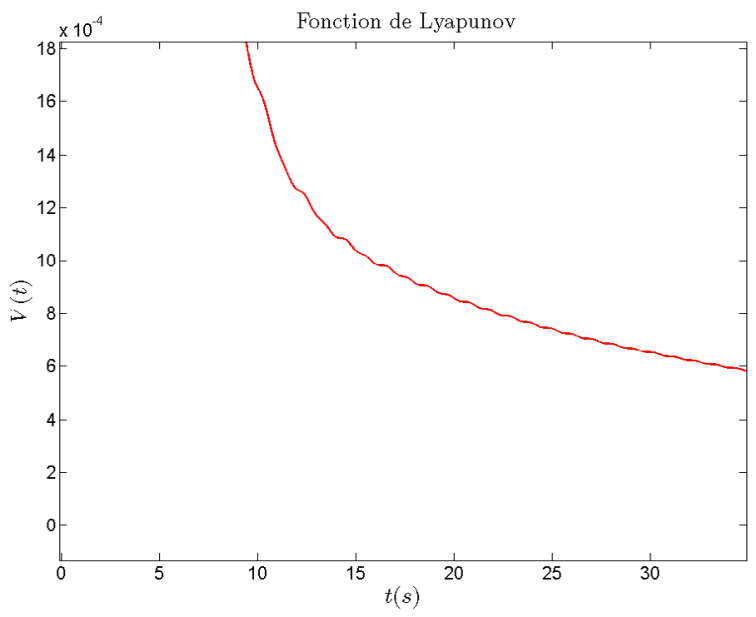

Figure 14. Slider point stabilization, $P_{\text {ref }}=\left(x_{\text {ref }}=0.5, y_{\text {ref }}=0.5, \psi_{\text {ref }}=-0.75\right), P_{\text {init }}=$ $\left(x_{\text {init }}=-1.6, y_{\text {init }}=0, \psi_{\text {init }}=-1.218\right)$, gains are $k_{1}=10, k_{3}=3, k_{4}=15, K_{1}=1, K=1$, $\omega=3$. (a) Slider trajectory on the $x / y$ plane, (b) associated Lyapunov function (4.70).
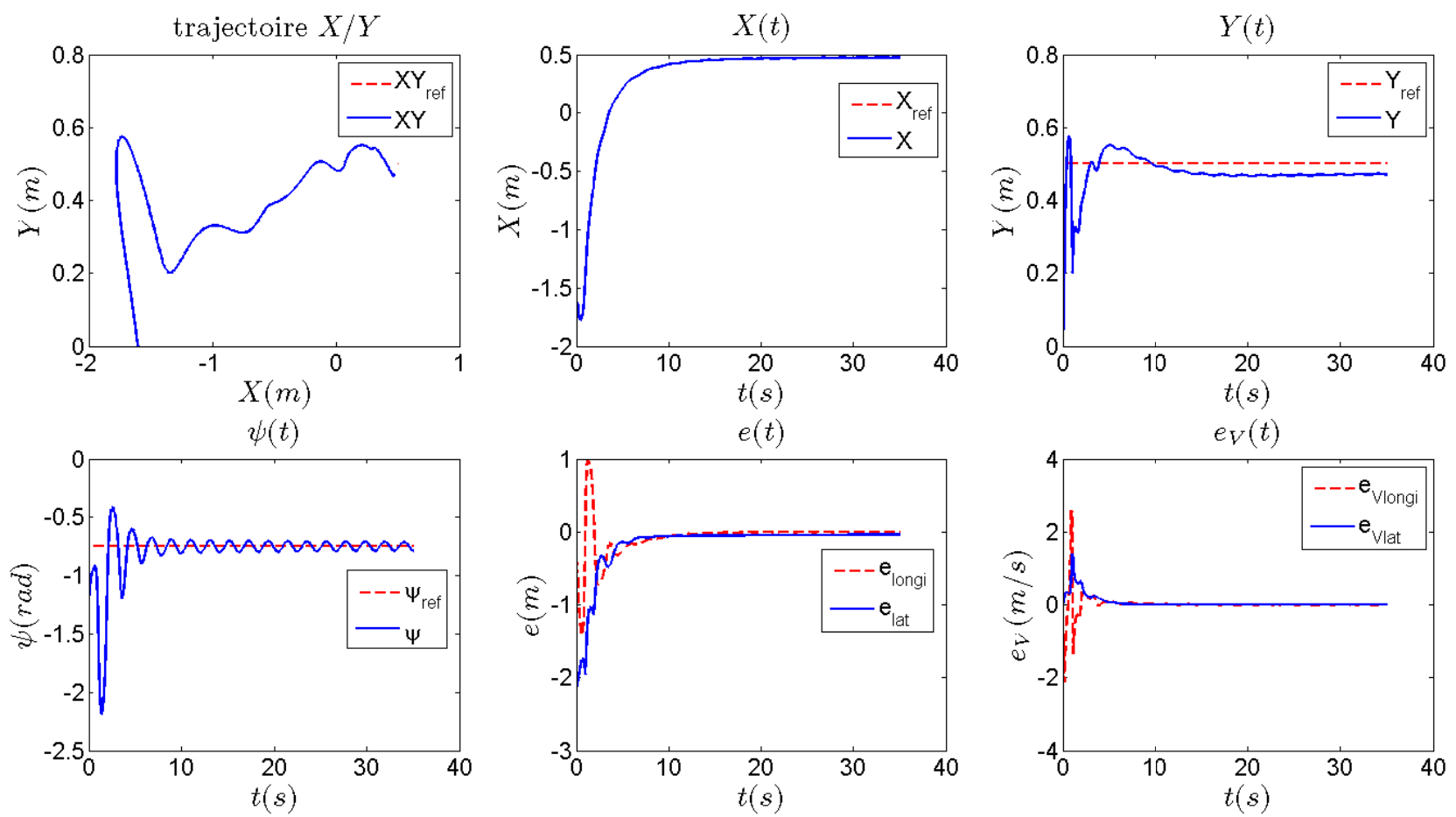

Figure 15. Slider point stabilization, $P_{\text {ref }}=\left(x_{\text {ref }}=0.5, y_{\text {ref }}=0.5, \psi_{\text {ref }}=-0.75\right), P_{\text {init }}=$ $\left(x_{\text {init }}=-1.6, y_{\text {init }}=0, \psi_{\text {init }}=-1.218\right)$, gains are $k_{1}=10, k_{3}=3, k_{4}=15, K_{1}=1, K=1$, $\omega=3$. 
Remark 4.15. At that step, $\dot{\psi}$ is considered as a virtual control input. Using backstepping arguments, it is possible to derive the expression of the physical control input $u_{2}$ from system's dynamics (4.53). For details (see [47], Chap. 4).

Remark 4.16. This control design, could be, in some sense, compared to the "phantom tracking method" developed by Coron in [12].

\section{Conclusion AND PERspeCtives}

It has been shown in the present paper that the unicycle dynamics shares similarities with the slider dynamics. Their common structure makes possible a comparison between these two systems in several fields of interest, such as stabilizability and controllability properties. Moreover, Brockett's necessary condition is not satisfied for both systems and therefore limits the use of continuous state feedback laws. Indeed, those control laws can stabilize neither unicycle systems nor sliders at a fixed point. In this paper, we have elaborated time-varying control laws stabilizing unicycle robots or sliders at a fixed point with a chosen reference orientation, and flatness-based feedback laws to track exciting reference trajectories. Therefore, a switching control strategy can be applied to track moving reference trajectories and ensure final stabilization at rest equilibrium points.

Let us point out that, in our study, the time-varying control law used for the slider fixed point stabilization can be extended to posture tracking of any reference trajectory. In that case, it has been shown in [47] that the convergence is practical and ensured in a neighborhood of the reference trajectory. The size of this neighborhood is highly dependent on the gains used in the control law. For non admissible trajectory, this result is not surprising but one can ask about admissible trajectories. Unfortunately, it has been demonstrated in [28] that universal stabilizers, even in the case of time-varying control laws, cannot stabilize systems like unicycles or sliders for both point stabilization and exciting reference trajectories. This issue was briefly introduced in [47]. It could be of interest to put this time-varying control law into perspective with regards to the results from [28].

\section{REFERENCES}

[1] B. d'Andréa-Novel and M. De Lara, Control Theory for Engineers. Springer (2013).

[2] B. d'Andréa-Novel, G. Bastin and G. Campion, Dynamic feedback linearization of non holonomic wheeled mobile robots. Proc. of the IEEE Conference on Robotics and Automation. Nice (1992) 2527-2532.

[3] B. d'Andréa-Novel, G. Campion and G. Bastin, Control of Non holonomic Wheeled Mobile Robots by State Feedback Linearization. Int. J. Robot. Res. 14 (1995) 543-559.

[4] E. Aranda-Bricaire, C.H. Moog and J.B. Pomet, A linear algebraic framework for dynamic feedback linearization. IEEE Transactions on Automatic Control 40 (1995) 127-132.

[5] R.W. Brockett, Asymptotic stability and feedback stabilization, Differential geometric control theory (Houghton, Mich., 1982). Vol. 27 of Progr. Math. Birkhauser, Boston, Boston, MA (1983) 181-191. MR 708502 (85e:93034).

[6] G. Campion, B. d'Andréa-Novel and G. Bastin, Modelling and state feedback control of non holonomic mechanical systems. Proc. of the 30th IEEE CDC (1991) 1184-1189.

[7] C. Canudas de Wit, B. Siciliano and G. Bastin, The ZODIAC, Theory of Robot Control. Springer, 1st edition (1997).

[8] B. Charlet, Sur quelques problèmes de stabilisation robuste des systèmes non linéaires. Ph.D. thesis, École Nationale Supérieure des Mines de Paris (1989).

[9] B. Charlet, J. Lévine and R. Marino, On dynamic feedback linearization. Syst. Control Lett. 13 (1989) $143-151$.

[10] J.-M. Coron, Global asymptotic stabilization for controllable systems without drift. Math. Control Signal Syst. 5 (1992) $295-312$.

[11] J.-M. Coron, On the stabilization in finite time of locally controllable systems by means of continuous time-varying feedback law. SIAM J. Control Optim. 33 (1995) 804-833.

[12] J.-M. Coron, Phantom tracking method, homogeneity and rapid stabilization. Math. Control Related Fields 3 (2013) 303-322.

[13] J.-M. Coron, Control and Nonlinearity. Vol. 136 of Mathematical Surveys and Monographs. American Mathematical Society (2007).

[14] J.-M. Coron and B. d'Andréa-Novel, Smooth stabilizing time-varying control laws for a class of nonlinear systems. Application to mobile robots. Proc. of the NOLCOS Conference. Bordeaux (1992) 649-654.

[15] J. Descusse and C.H. Moog, Decoupling with dynamic compensation for strong invertible affine nonlinear systems. Int. J. Control 42 (1985) 1387-1398.

[16] R. Lozano, Objets volants miniatures. Hermes Science, Lavoisier (2007).

[17] I. Fantoni, R. Lozano, F. Mazenc and K.Y. Pettersen, Stabilization of a nonlinear under-actuated hovercraft. Proc. of the 38th IEEE CDC (1999). 
[18] M. Fliess, J. Lévine, P. Martin and P. Rouchon, On differentially flat nonlinear systems. Proc. of the 2nd IFAC NOLCOS Symposium. Bordeaux (1992) 408-412.

[19] M. Fliess, J. Lévine, P. Martin and P. Rouchon, Flatness and defect of nonlinear systems: introductory theory and examples. Int. J. Control 61 (1995) 1327-1361.

[20] W. Hahn, Stability of motion. Springer, Berlin, Heidelberg, New-York (1967).

[21] A. Isidori, Nonlinear Control Systems. Springer-Verlag, 3rd Edition, London (1995).

[22] Y. Kanayama, Y. Kimura, F. Miyazaki and T. Noguchi, A stable tracking control method for an autonomous mobile robot. Proc. of the IEEE Int. Conf. Robot. Automat. (1990) 384-389.

[23] H.K. Khalil, Nonlinear systems. Prentice Hall, 2nd Edition (1995).

[24] G. Kern, Uniform controllability of a class of linear time-varying systems. IEEE Trans. Automat. Control 27 (1982) $208-210$.

[25] P. Kokotović and H. Sussmann, A positive real condition for global stabilization of nonlinear systems. Syst. Control Lett. 13 (1989) 125-133.

[26] I. Kolmanovsky and N.H. McClamroch, Developments in non holonomic control problems. IEEE Control Syst. 15 (1995) $20-36$.

[27] E. Lefeber, K.Y. Pettersen and H. Nijmeijer, Tracking control of an under-actuated ship. IEEE Trans. Control System Technol. 11 (2003) 52-61.

[28] D.A. Lizárraga. Obstructions to the Existence of Universal Stabilizers for Smooth Control Systems. Math. Control Signals Syst 16 (2004) 255-277.

[29] A. Micaelli, B. d'Andréa-Novel and B. Thuilot, Modeling and asymptotic stabilization of mobile robots equipped with two or more steering wheels. Proc. of ICARCV'92. Singapour (1992).

[30] P. Morin and C. Samson, Control of nonlinear chained systems. From the Routh-Hurwitz stability criterion to time-varying exponential stabilizers. Rapport INRIA 3126 (1997).

[31] P. Morin and C. Samson, Control of nonlinear chained systems. From the Routh-Hurwitz stability criterion to time-varying exponential stabilizers. Proc. of the 36th IEEE CDC 1 (1997) 618-623.

[32] P. Morin and C. Samson, Control of under-actuated mechanical systems by the transverse function approach. In 44 th IEEE Conference on Decision and Control, 2005 and 2005 European Control Conference. CDC-ECC '05 (2005) 7508-7513.

[33] P. Morin and C. Samson, Transverse functions on special orthogonal groups for vector fields satisfying the LARC at the order one. In Proc. of the 48th IEEE Conference on Decision and Control, 2009 held jointly with the 200928 th Chinese Control Conference. CDC/CCC (2009) 7472-7477.

[34] R.M. Murray and S.S. Sastry, Steering non holonomic systems in chained form. In vol. 2, Proc. of the 30th IEEE CDC (1991) $1121-1126$.

[35] R.M. Murray, Z. Li, S. Shankar Sastry and S. Shankara Sastry, A mathematical introduction to robotic manipulation. CRC press (1994).

[36] K.Y. Pettersen and O. Egeland, Exponential stabilization of an under-actuated surface vessel. Proc. of the 35th IEEE CDC (1996) 967-972.

[37] K.Y. Pettersen and O. Egeland, Robust control of an under-actuated surface vessel with thruster dynamics. Proc. of the ACC (1997).

[38] K.Y. Pettersen and H. Nijmeijer, Global practical stabilization and tracking for an under-actuated ship - a combined averaging and backstepping approach. Model. Identif. Control 20 (1999) 189-200.

[39] J.-B. Pomet, B. Thuilot, G. Bastin and G. Campion, A hybrid strategy for the feedback stabilization of non holonomic mobile robots, Proc. of the IEEE Conf. on Robotics and Automation. Nice (1992) 129-134.

[40] L. Praly, B. d'Andréa-Novel and J.-M. Coron, Lyapunov design of stabilizing controllers for cascaded systems. In vol. 36, IEEE Trans. Automat. Control (1991) 10.

[41] M. Reyhanoglu. Control and stabilization of an under-actuated surface vessel. In vol. 3, Proc. of the 35th IEEE Conf. Decision Control (1996) 2371-2376.

[42] P. Rouchon, Necessary condition and genericity of dynamic feedback linearization. J. Math. Systems Estim. Control (1994) $345-358$.

[43] C. Samson, Path following and time-varying feedback stabilization of a wheeled mobile robot. In vol. 13, Proc. of the International Conference on Advanced Robotics and Computer Vision. Singapour (1992) 1.1-1.5.

[44] C. Samson, Control of chained systems. Application to path following and time-varying feedback stabilization of mobile robots. IEEE Trans. Automat. Control 40 (1995) 64-77.

[45] C. Samson, K. Ait-Abderrahim, Mobile robot control. Part 1: feedback control of non holonomic wheeled cart in cartesian space. Rapport de recherche RR-1288, INRIA (1990).

[46] R. Sepulchre, M. Janković, P. Kokotović, Constructive Nonlinear Control. Springer (1997).

[47] S. Thorel, Conception et réalisation d'un drone hybride sol/air autonome. Ph.D. thesis, MINES ParisTech (2014).

[48] S. Thorel and B. d'Andréa-Novel, Hybrid terrestrial and aerial quadrotor control. Proc. of the 19th IFAC World Congress. South Africa (2014).

[49] S. Thorel and B. d'Andréa-Novel, Practical identification and flatness based control of a terrestrial quadrotor. Proc. of the IROS conference. Chicago (2014).

[50] B. Thuilot, B. d'Andréa-Novel and A. Micaelli, Modeling and Feedback Control of Mobile Robots Equipped with Several Steering Wheels. IEEE Trans. Robot. Automat. 12 (1996) 375-390.

[51] J. Tsinias, Sufficient Lyapunov-like conditions for stabilization. Math. Control Signals Syst. 2 (1989) $343-357$. 